arXiv:0810.0937 [hep-th]

YITP-08-91

\title{
AdS Vacua, Attractor Mechanism and Generalized Geometries
}

\author{
Tetsuji KimURA \\ Yukawa Institute for Theoretical Physics, Kyoto University \\ Sakyo-ku, Kyoto 606-8502, Japan \\ tetsuji@yukawa.kyoto-u.ac.jp
}

\begin{abstract}
We consider flux vacua attractor equations in type IIA string theory compactified on generalized geometries with orientifold projections. The four-dimensional $\mathcal{N}=1$ superpotential in this compactification can be written as the sum of the Ramond-Ramond superpotential and a term described by (non)geometric flux charges. We exhibit a simple model in which supersymmetric AdS and Minkowski solutions are classified by means of discriminants of the two superpotentials. We further study various configurations without Ramond-Ramond flux charges. In this case we find supersymmetric AdS vacua both in the case of compactifications on generalized geometries with $S U(3) \times S U(3)$ structures and on manifolds with an $S U(3)$-structure without nongeometric flux charges. In the latter case, we have to introduce correction terms into the prepotential in order to realize consistent vacua.
\end{abstract}




\section{Introduction}

In the search of a model describing realistic physics, many string compactification scenarios have been developed and studied. One remarkable success is that of compactification on a Calabi-Yau three-fold [1], which gives a supersymmetric Minkowski vacuum in four-dimensional effective gauge theory. However, this configuration is insufficient as a candidate of a realistic physical vacuum in string theory because it assumes four significant simplifications: constant dilaton, vanishing $H$-flux, flat Minkowski space, and $\mathcal{N}=1$ supersymmetry. Once some of these assumptions are relaxed, a rich structure emerges in the compactified space, which also affects the four-dimensional effective theory. In particular, on a six-dimensional internal space with $S U(3)$-structure [2], a non-vanishing NS-NS three-form flux $H$ yields torsion. Such geometries have been investigated both in mathematics [3] and in string theory [4]. Furthermore, Hitchin's generalized geometry [5] contains information about the $S U(3)$-structure manifold with torsion, and provides a powerful technique in the investigation of four-dimensional $\mathcal{N}=2$ and $\mathcal{N}=1$ supergravity theories (see [6-16] and references therein).

Four-dimensional $\mathcal{N}=2$ (gauged) supergravity is not only dynamical but also controllable by two moduli spaces, namely a special geometry and a quaternionic geometry [17]. Generalized geometry also has two moduli spaces described as special geometries. Due to the existence of these moduli spaces, one can embed the four-dimensional $\mathcal{N}=2$ supergravity into type IIA (or IIB) string theory compactified on a generalized geometry. Various functions in four-dimensional spacetime such as the Kähler potential and the superpotential are written in terms of the prepotentials on the moduli spaces and of various fluxes such as geometric fluxes and form fluxes on the internal space. The most generic forms of these functions are described by Graña, Louis and Waldram [7], and Benmachiche and Grimm demonstrated a consistent procedure to truncate the model from $\mathcal{N}=2$ to $\mathcal{N}=1$ supersymmetry via an orientifold projection on generalized geometry [11]. Graña, Minasian, Petrini and Tomasiello performed a clever application of "scanning" technique to $\mathcal{N}=1$ vacua on parallelizable nilmanifolds and solvmanifolds described as generalized geometries with a single $S U(3)$-structure [12]. In [15] Cassani and Bilal carefully investigated the Kähler potential and superpotential in four-dimensional $\mathcal{N}=1$ supergravity obtained from type IIA string theory compactified on generalized geometry with $S U(3) \times S U(3)$ structures.

String compactifications in the presence of fluxes also give rise to non-abelian gauge symmetries in four-dimensional models, whereas the compactification on a Calabi-Yau space does not. In order to realize such a gauge symmetry, one introduces a twist in the (generalized) Scherk-Schwarz compactification procedure [18-20], possibly on some extended internal space, which yields "nongeometric" fluxes $[21,22]$ as well as geometric fluxes. One candidate for the internal space is generalized geometry with $S U(3) \times S U(3)$ structures $[7,16]$. Other techniques, such as compactification in the framework of doubled space formalism $[23,24]$, have also been investigated to explain nongeometric fluxes as arising from string dualities. 
The aim of this paper is to realize consistent supersymmetric Anti-de Sitter (AdS) vacua as well as Minkowski vacua in type IIA theory with or without Ramond-Ramond fluxes [25-30]. One way to obtain such vacua is by use of the attractor mechanism. Originally the attractor mechanism was developed in the analysis of the entropy of extremal (non-)BPS black holes in type II theories [31-37]. This mechanism has been applied in the search of flux vacua [38-44], since the functions and equations in both black holes and flux vacua frameworks are quite similar. In the black hole attractors one focuses on the black hole potential [33], while in the flux vacua attractors one studies the scalar potential in $\mathcal{N}=1$ supergravity [38]. In both cases one investigates extreme points (called attractor points) by evaluating the potentials, which are expressed in terms of the $\mathcal{N}=1$ superpotential. The scalar potential is described in terms of the Kähler potential $K$ and the superpotential $\mathcal{W}$ as

$$
V=\mathrm{e}^{K}\left(K^{M \bar{N}} D_{M} \mathcal{W} D_{\bar{N}} \overline{\mathcal{W}}-3|\mathcal{W}|^{2}\right)+\frac{1}{2}(\operatorname{Re} f)_{\hat{a} \hat{b}} D^{\hat{a}} \bar{D}^{\hat{b}} \equiv V_{\mathcal{W}}+V_{D}
$$

where $D_{M}$ is the Kähler covariant derivative with respect to complex scalar fields $\phi^{M}$, defined as $D_{M} \mathcal{W} \equiv\left(\partial_{M}+\partial_{M} K\right) \mathcal{W}$, and $K_{M \bar{N}}=\partial_{M} \partial_{\bar{N}} K(\phi, \bar{\phi})$ is the Kähler metric. The $\phi^{M}$ collectively denote all complex scalars in all chiral multiplets present in the $\mathcal{N}=1$ theory. The second term on the righthand side carries the D-terms $D^{\hat{a}}$ which belong to vector multiplets. The attractor point is defined by the equation $\phi^{M}=\phi_{*}^{M}$ satisfying $\partial V /\left.\partial \phi^{M}\right|_{*}=0$.

This paper is structured as follows: In section 2 we write down the scalar potential and its derivatives in four-dimensional $\mathcal{N}=1$ supergravity. We evaluate the derivatives of the scalar potential, which are called attractor equations. In order to make our discussion clear, we restrict the prepotential governing the superpotential to a simple form. In sections $3,4,5$ and 6 , we find flux vacua attractors in various examples. In section 3 we analyze a model in which Ramond-Ramond fluxes as well as (non)geometric fluxes are introduced. In this analysis two discriminants of the superpotential play central roles in the classification of supersymmetric vacua, where the discriminants are written in terms of flux charges. If the discriminants are positive, we obtain a supersymmetric AdS vacuum whose cosmological constant is governed by (the square root of) a discriminant of the superpotential. If the discriminants are negative, we obtain a supersymmetric Minkowski vacuum. In section 4 we analyze a different model in which Ramond-Ramond flux charges are absent, whereas nongeometric flux charges are present. There we again obtain a supersymmetric AdS vacuum. In section 5 we study other models which carry only geometric flux charges, where we find neither supersymmetric nor nonsupersymmetric solutions if the prepotential is expressed only in terms of the intersection number, as in the case of Calabi-Yau compactification in the large volume limit. In section 6 we introduce correction terms to the prepotential in order to find supersymmetric vacua in the presence of geometric flux charges, but without nongeometric and Ramond-Ramond flux charges. We can interpret each of the models in this section as coming from heterotic string theory compactified on a torsionful manifold with a single $S U(3)$-structure. Section 7 is devoted to the summary and discussions. To streamline the arguments, brief derivations of the functions in section 2 are included in appendices. 


\section{Analysis of scalar potential}

In this section we analyze the scalar potential (1.1). We start with type IIA string theory compactified on generalized geometry with $S U(3) \times S U(3)$ structures. This compactification yields the superpotential $\mathcal{W}$, the Kähler potential $K$, the dilaton $\varphi$ and the D-terms $D^{\hat{A}}$ in four-dimensional spacetime. Their explicit forms are

$$
\begin{aligned}
\mathcal{W} & =-\frac{i}{4 \bar{a} b}\left[X^{\check{A}}\left(e_{\mathrm{RR} \check{A}}-U^{\check{I}} e_{\check{I} \check{A}}+\widetilde{U}_{\hat{I}} m_{\check{A}}^{\hat{I}}\right)-\mathcal{F}_{\check{A}}\left(m_{\mathrm{RR}}^{\check{A}}+U^{\check{I}} p_{\check{I}}^{\check{A}}-\widetilde{U}_{\hat{I}} q^{\hat{I} \check{A}}\right)\right], \\
K & =K_{+}+4 \varphi \\
K_{+} & =-\log i\left(\bar{X}^{\check{A}} \mathcal{F}_{\check{A}}-X^{\check{A}} \overline{\mathcal{F}}_{\check{A}}\right), \\
\mathrm{e}^{-2 \varphi} & =\frac{1}{2}\left[\operatorname{Im}\left(\mathcal{C} Z^{\check{I}}\right) \operatorname{Re}\left(\mathcal{C} \mathcal{G}_{\check{I}}\right)-\operatorname{Re}\left(\mathcal{C} Z^{\hat{I}}\right) \operatorname{Im}\left(\mathcal{C} \mathcal{G}_{\hat{I}}\right)\right], \\
D^{\hat{A}} & =\mathrm{e}^{2 \varphi}\left[(\operatorname{Im} \mathcal{N})^{-1}\right]^{\hat{A} \hat{B}}\left\{\operatorname{Re}\left(\mathcal{C} Z^{\hat{I}}\right)\left[e_{\hat{I} \hat{B}}+\mathcal{N}_{\hat{B} \hat{C}} p_{\hat{I}}^{\hat{C}}\right]-\operatorname{Re}\left(\mathcal{C} \mathcal{G}_{\check{I}}\right)\left[m_{\hat{B}}^{\check{I}}+\mathcal{N}_{\hat{B} \hat{C}} q^{\check{I} \hat{C}}\right]\right\} .
\end{aligned}
$$

Here we used notation and conventions in $[7,12,15,45]$. We summarize derivations of the above functions in the appendices.

Let us search an extreme point of the scalar potential given by $\partial_{P} V=0$ with respect to holomorphic variables. The first derivatives are written as

$$
\begin{aligned}
& \partial_{P} V_{\mathcal{W}}=\mathrm{e}^{K}\left\{K^{M \bar{N}} D_{P} D_{M} \mathcal{W} D_{\bar{N}} \overline{\mathcal{W}}+\partial_{P} K^{M \bar{N}} D_{M} \mathcal{W} D_{\bar{N}} \overline{\mathcal{W}}-2 \overline{\mathcal{W}} D_{P} \mathcal{W}\right\}, \\
& \partial_{P} V_{D}=\frac{1}{2} \partial_{P}(\operatorname{Re} f)_{\hat{a} \hat{b}} D^{\hat{a}} \bar{D}^{\hat{b}}+\frac{1}{2}(\operatorname{Re} f)_{\hat{a} \hat{b}} \partial_{P} D^{\hat{a}} \bar{D}^{\hat{b}}+\frac{1}{2}(\operatorname{Re} f)_{\hat{a} \hat{b}} D^{\hat{a}} \partial_{P} \bar{D}^{\hat{b}},
\end{aligned}
$$

where we used $\partial_{P} \overline{\mathcal{W}}=0$ and a set of equations:

$$
\begin{aligned}
\overline{\mathcal{W}} \partial_{P} \mathcal{W} & =\overline{\mathcal{W}} D_{P} \mathcal{W}-\partial_{P} K|\mathcal{W}|^{2} \\
D_{P} D_{M} \mathcal{W} & =\partial_{P} D_{M} \mathcal{W}+\partial_{P} K D_{M} \mathcal{W}, \\
D_{P} D_{\bar{N}} \overline{\mathcal{W}} & =K_{P \bar{N}} \overline{\mathcal{W}} .
\end{aligned}
$$

The Kähler covariant derivative is defined in terms of the Kähler potential $K=K_{+}+4 \varphi$. This does not inherit the property of the special Kähler geometry of local type.

We look for a solution which satisfies $\partial_{P} V_{\mathcal{W}}=0$ and $\partial_{P} V_{D}=0$. This is realized when the supersymmetry condition $D_{P} \mathcal{W}=0$ is satisfied. The equation $D_{P} \mathcal{W}=0$ is called the attractor equation in supersymmetric attractor mechanism. The holomorphic scalar fields are described by

$$
\begin{aligned}
t^{\check{a}} & =\frac{X^{\check{a}}}{X^{0}}=b^{\check{a}}+i v^{\check{a}}, \quad U^{\check{I}}=\xi^{\check{I}}+i \operatorname{Im}\left(\mathcal{C} Z^{\check{I}}\right), \quad \widetilde{U}_{\hat{I}}=\widetilde{\xi}_{\hat{I}}+i \operatorname{Im}\left(\mathcal{C} \mathcal{G}_{\hat{I}}\right), \\
I & =0,1, \ldots, b^{-} \quad \check{I}=0,1, \ldots, \check{b}^{-}, \quad \hat{I}=1, \ldots, \hat{b}^{-}, \quad \hat{b}^{-} \equiv b^{-}-\check{b}^{-} .
\end{aligned}
$$

Since $Z^{0}$ is compensated by the four-dimensional dilaton $\varphi$ via the combination $\mathcal{C} Z^{0}[15], U^{0}=$ $\xi^{0}+i \operatorname{Im}\left(\mathcal{C} Z^{0}\right)$ is dynamical. 
In order to extract significant property of vacua, it is much instructive to restrict the prepotentials. Precisely speaking, we reduce the prepotential $\mathcal{F}$ on $\mathcal{M}_{+}$and the number of degrees of freedom in the moduli space $\mathcal{M}_{-}$. Here we set the prepotential $\mathcal{F}[15,45]$ in the following form:

$$
\mathcal{F} \equiv D_{a b c} \frac{X^{a} X^{b} X^{c}}{X^{0}}, \quad D_{a b c}=-\frac{1}{6} \mathcal{K}_{a b c} .
$$

We should keep in mind that the expression (2.5) implies that all $\alpha^{\prime}$ corrections are neglected. In Calabi-Yau compactification this setting is usual, while quite restricted in the case of compactifications on generalized geometries. We will discuss this issue in sections 5 and 6 . We also restrict another moduli space $\mathcal{M}_{-}$. For simplicity, we reduce the number of moduli. We set $\check{I}=\{0\}$. This means that the remaining dynamical field is $U^{0}$, and we truncate out all of $U^{\breve{i}}$ and $\widetilde{U}_{\hat{I}}$. From now on we abbreviate $U^{0}$ to $U$. As far as we concern this reduction, the constraints (B.13) are trivial.

\subsection{Derivatives of superpotential}

The superpotential (2.1a) is governed by the Ramond-Ramond flux charges and the (non)geometric flux charges. Its explicit form is

$$
\begin{gathered}
\mathcal{W}=\mathcal{W}^{\mathrm{RR}}+U \mathcal{W}^{\mathcal{Q}}, \\
\mathcal{W}^{\mathrm{RR}} \equiv-\frac{i}{4 \bar{a} b}\left(X^{\check{A}} e_{\mathrm{RR} \check{A}}-\mathcal{F}_{\check{A}} m_{\mathrm{RR}}^{\check{A}}\right), \quad \mathcal{W}^{\mathcal{Q}} \equiv \frac{i}{4 \bar{a} b}\left(X^{\check{A}} e_{0 \check{A}}+\mathcal{F}_{\check{A}} p_{0}^{\check{A}}\right) .
\end{gathered}
$$

We refer to $\mathcal{W}^{\mathrm{RR}}$ as the Ramond-Ramond flux superpotential, and to $\mathcal{W}^{\mathrm{RR}}$ as the (non)geometric flux superpotential. The Kähler covariant derivatives acting on the superpotential are

$$
\begin{aligned}
& D_{\check{a}} \mathcal{W}=D_{\check{a}} \mathcal{W}^{\mathrm{RR}}+U D_{\check{a}} \mathcal{W}^{\mathcal{Q}}, \\
& D_{U} \mathcal{W}=\frac{i}{\operatorname{Im} U}\left(\mathcal{W}^{\mathrm{RR}}+\operatorname{Re} U \mathcal{W}^{\mathcal{Q}}\right) .
\end{aligned}
$$

We study the second Kähler covariant derivatives of the superpotential $D_{M} D_{N} \mathcal{W}=\partial_{M} D_{N} \mathcal{W}+$ $\partial_{M} K D_{N} \mathcal{W}$. The explicit forms are

$$
\begin{aligned}
D_{\check{b}} D_{\check{a}} \mathcal{W} & =D_{\check{b}} D_{\check{a}} \mathcal{W}^{\mathrm{RR}}+U D_{\check{b}} D_{\check{a}} \mathcal{W}^{\mathcal{Q}}=i C_{\check{a} \check{b} \check{c}}\left(K_{+}\right)^{\check{c} \breve{d}}\left(D_{\breve{d}} \overline{\mathcal{W}}^{\mathrm{RR}}+U D_{\breve{d}} \overline{\mathcal{W}}^{\mathcal{Q}}\right), \\
D_{U} D_{\check{a}} \mathcal{W} & =D_{\check{a}} \mathcal{W}^{\mathcal{Q}}+\frac{i}{\operatorname{Im} U} D_{\check{a}} \mathcal{W}, \\
D_{U} D_{U} \mathcal{W} & =\frac{i}{2 \operatorname{Im} U}\left(3 D_{U} \mathcal{W}+\mathcal{W}^{\mathcal{Q}}\right) .
\end{aligned}
$$

Since $t^{a}$ is independent of $U$, we can use a formula $D_{a} D_{b}\left(\mathrm{e}^{\frac{K_{+}}{2}} \mathcal{W}\right)=i C_{a b c}\left(K_{+}\right)^{c \bar{d}} D_{\bar{d}}\left(\mathrm{e}^{\frac{K_{+}}{2}} \overline{\mathcal{W}}\right)$. As far as we concern the system with the prepotential (2.5), the imaginary part of $U$ does not vanish, otherwise the Kähler metric $K_{U \bar{U}}$ and the curvature tensor $R^{U}{ }_{U U}$ become singular. 
Now we are ready to evaluate the extreme point of $V_{\mathcal{W}}$. Due to the equations (2.7), we obtain a set of differential equations and an algebraic equation at the extreme point $\left(t^{\check{a}}, U\right)=\left(t_{*}^{\check{a}}, U_{*}\right)$ :

$$
\begin{array}{rlrlrl}
\left.D_{\check{a}} \mathcal{W}\right|_{*} & =0 & \rightarrow & \left.D_{\check{a}} \mathcal{W}^{\mathrm{RR}}\right|_{*} & =-\left.U_{*} D_{\check{a}} \mathcal{W}^{\mathcal{Q}}\right|_{*}, \\
\left.D_{U} \mathcal{W}\right|_{*}=0 & \rightarrow & \mathcal{W}_{*}^{\mathrm{RR}} & =-\operatorname{Re} U_{*} \mathcal{W}_{*}^{\mathcal{Q}} .
\end{array}
$$

If both $D_{\breve{a}} \mathcal{W}^{\mathrm{RR}}$ and $D_{\check{a}} \mathcal{W}^{\mathcal{Q}}$ vanish to satisfy (2.9a) while $\mathcal{W}^{\mathrm{RR}}$ and $\mathcal{W}^{\mathcal{Q}}$ do not vanish, we obtain flux vacua attractor equations. These are exactly the same equations in the black hole attractors in type IIA theory [32-34]. On the other hand, if we can take $\left.D_{\check{a}} \mathcal{W}^{\mathrm{RR}}\right|_{*} \neq 0$ with $\left.D_{\check{a}} \mathcal{W}\right|_{*}=0$, we can employ the non-supersymmetric black hole analyses in finding supersymmetric flux vacua. The discussions of a classification of (non)supersymmetric vacua can be seen in [43].

\subsection{Derivatives of D-term}

Investigation of the D-term (2.1e) is interesting, because its non-trivial value breaks supersymmetry. It is known that

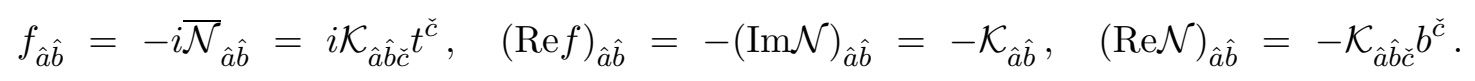

Here let us write a concrete form

$$
D^{\hat{a}}=-\frac{2}{\operatorname{Im} U}\left[(\operatorname{Im} \mathcal{N})^{-1}\right]^{\hat{a} \hat{b}}\left(m_{\hat{b}}^{0}+\mathcal{N}_{\hat{b} \hat{c}} q^{0 \hat{c}}\right) .
$$

Note that the indices $\hat{A}$ are reduced to $\hat{a}$, since the graviphoton $A_{\mu}^{0}$ is always truncated out. The intersection number $\mathcal{K}_{\hat{a} \hat{b} \check{c}}=\int_{\mathcal{M}} \omega_{\hat{a}} \wedge \omega_{\hat{b}} \wedge \omega_{\check{c}}$ is a constant. It is useful to introduce an inverse $\mathcal{K}^{\hat{a} \hat{b}}$ which satisfies a relation $\mathcal{K}^{\hat{a} \hat{b}} \mathcal{K}_{\hat{b} \hat{c}}=\delta_{\hat{c}}^{\hat{a}}$. The D-term and the potential can be rewritten as

$$
\mu_{\hat{a}} \equiv-(\operatorname{Im} \mathcal{N})_{\hat{a} \hat{b}} D^{\hat{b}}=\frac{2}{\operatorname{Im} U}\left(m_{\hat{a}}^{0}-\mathcal{K}_{\hat{a} \hat{b} \check{c}} \bar{t}^{\check{c}} q^{0 \hat{b}}\right), \quad V_{D}=-\frac{1}{2} \mathcal{K}^{\hat{a} \hat{b}} \mu_{\hat{a}} \bar{\mu}_{\hat{b}} .
$$

The first derivative $\partial_{P} V_{D}$ is written as $\partial_{P} V_{D}=-\frac{1}{2} \partial_{P} \mathcal{K}^{\hat{a} \hat{b}} \mu_{\hat{a}} \bar{\mu}_{\hat{b}}-\frac{1}{2} \mathcal{K}^{\hat{a} \hat{b}} \partial_{P} \mu_{\hat{a}} \bar{\mu}_{\hat{b}}-\frac{1}{2} \mathcal{K}^{\hat{a} \hat{b}} \mu_{\hat{a}} \partial_{P} \bar{\mu}_{\hat{b}}$. The derivatives depend only on the complex variables $t^{\check{a}}=b^{\check{a}}+i v^{\check{a}}$ :

$$
\partial_{\check{c}} \mathcal{K}_{\hat{a} \hat{b}}=\mathcal{K}_{\hat{a} \hat{b} \check{d}} \frac{\partial v^{\check{d}}}{\partial t^{\check{c}}}=-i \mathcal{K}_{\hat{a} \hat{b} \check{c}}, \quad \partial_{\check{c}} \mathcal{N}_{\hat{a} \hat{b}}=-\mathcal{K}_{\hat{a} \hat{b} \hat{d}} \frac{\partial \bar{t}^{\check{d}}}{\partial t^{\check{c}}}=0, \quad \partial_{\check{c}} \overline{\mathcal{N}}_{\hat{a} \hat{b}}=-\mathcal{K}_{\hat{a} \hat{b} \check{c}}
$$

We also study the first derivatives of $\mu_{\hat{a}}$ :

$$
\partial_{\hat{b}} \mu_{\hat{a}}=0, \quad \partial_{\hat{b}} \bar{\mu}_{\hat{a}}=-\frac{2}{\operatorname{Im} U} \mathcal{K}_{\hat{a} \hat{c} \check{b}} q^{0 \hat{c}}, \quad \partial_{U} \mu_{\hat{a}}=\frac{i}{2 \operatorname{Im} U} \mu_{\hat{a}}, \quad \partial_{U} \bar{\mu}_{\hat{a}}=\frac{i}{2 \operatorname{Im} U} \bar{\mu}_{\hat{a}} .
$$

Then we obtain

$$
\partial_{\check{c}} V_{D}=-\frac{i}{2} \mathcal{K}^{\hat{a} \hat{e}} \mathcal{K}_{\check{c} \hat{d} \hat{e}} \mu_{\hat{a}}\left(\mathcal{K}^{\hat{b} \hat{d}_{\bar{b}}}+\frac{2 i}{\operatorname{Im} U} q^{0 \hat{d}}\right), \quad \partial_{U} V_{D}=-\frac{i}{4 \operatorname{Im} U} \mathcal{K}^{\hat{a} \hat{b}} \mu_{\hat{a}} \bar{\mu}_{\hat{b}}
$$


Since there are no contributions of $m_{\hat{a}}^{0}$ and $q^{0 \hat{a}}$ to the scalar potential $V_{\mathcal{W}}$, we can evaluate the extreme point of $V_{D}$ independently. If we consider the condition $\left.\partial_{P} V_{D}\right|_{*}=0$, we find that

$$
\left.\mu_{\hat{a}}\right|_{*}=0
$$

is the solution. This implies that the D-term contribution does not break supersymmetry and the scalar potential $V_{D}$ vanishes at the extreme point. Then it is enough to focus on the scalar potential $V_{\mathcal{W}}$ in order to analyze whether supersymmetry of the effective theory is broken or not.

\subsection{Search of (non)supersymmetric flux attractor vacua}

So far we specified the Kähler potential, the superpotential, the D-term and their derivatives. In next sections we study various simple examples which show intrinsic phenomena in supersymmetric flux vacua. First, we consider a setup which contains not only Ramond-Ramond fluxes but also (non)geometric fluxes. In this setup we obtain a simple but powerful rule to find supersymmetric vacua. Second, we study other cases in which the Ramond-Ramond flux charges are absent. More precisely we consider the following three cases: (i) No Ramond-Ramond flux charges in the presence of the nongeometric flux charges: (ii) No Ramond-Ramond flux charges in the absence of the nongeometric flux charges without any corrections in the prepotential (2.5): (iii) No Ramond-Ramond flux charges in the absence of the nongeometric flux charges with corrections in the prepotential. Indeed a generalized geometry with neither the Ramond-Ramond fluxes nor the nongeometric fluxes corresponds to an $S U(3)$-structure manifold in string flux compactification. We do not consider other situations that all the (non)geometric flux charges vanish while there exist non-zero Ramond-Ramond flux charges. These configurations are forbidden [25] because the Ramond-Ramond fluxes induce the non-zero valued NS-NS flux and some torsion classes.

\section{Example 1: a model with Ramond-Ramond flux charges}

\section{$3.1 \quad$ Strategy}

In section 2 we discussed the attractor equations $D_{P} \mathcal{W}=0$. Here we set $a=\bar{b} \mathrm{e}^{i \theta}$ and $|a|^{2}=|b|^{2}=\frac{1}{2}$ as in (D.1) via the $\mathrm{O} 6$ orientifold projection. An arbitrary parameter $\theta$ is absorbed in the phase of $a$ (or $b$ ) to set $2 \bar{a} b=-i$. We rescale all the flux charges by integer 2 without loss of generality. The scalar potential on the extreme point is given by

$$
\begin{gathered}
\left.D_{\check{a}} \mathcal{W}\right|_{*}=0,\left.\quad D_{U} \mathcal{W}\right|_{*}=0 \\
V_{*}=\mathrm{e}^{K}\left(K^{M \bar{N}} D_{M} \mathcal{W} D_{\bar{N}} \overline{\mathcal{W}}-3|\mathcal{W}|^{2}\right)_{*}-\left.\frac{1}{2} \mathcal{K}^{\hat{a} \hat{b}} \mu_{\hat{a}} \bar{\mu}_{\hat{b}}\right|_{*}=-3 \mathrm{e}^{K}\left|\mathcal{W}_{*}\right|^{2} .
\end{gathered}
$$


This gives a non-positive cosmological constant. The four-dimensional spacetime becomes a Minkowski space (if $\mathcal{W}_{*}=0$ ) or an $\operatorname{AdS}$ space (if $\mathcal{W}_{*} \neq 0$ ). Here let us consider a model governed by a single modulus $t^{\breve{a}} \equiv t$. Various functions are simplified:

$$
K_{+}=-\log \left(-i D(t-\bar{t})^{3}\right), \quad C_{t t t}=\frac{6 i}{(t-\bar{t})^{3}}
$$

where we set $D_{\check{a} \breve{b} \check{c}}=D$. The superpotentials $\mathcal{W}^{\mathrm{RR}}$ and $\mathcal{W}^{\mathcal{Q}}(2.6)$ are explicitly given by

$$
\begin{aligned}
\mathcal{W}^{\mathrm{RR}} & =X^{\check{A}} e_{\mathrm{RR} \check{A}}-\mathcal{F}_{\check{A}} m_{\mathrm{RR}}^{\check{A}}=e_{\mathrm{RR} 0}+e_{\mathrm{RR}} t-3 m_{\mathrm{RR}} t^{2}+m_{\mathrm{RR}}^{0} t^{3}, \\
\mathcal{W}^{\mathcal{Q}} & =-\left(X^{\check{A}} e_{0 \check{A}}+\mathcal{F}_{\check{A}} p_{0}{ }^{\check{A}}\right)=-e_{00}-e_{0} t-3 p_{0} t^{2}+p_{0}{ }^{0} t^{3} .
\end{aligned}
$$

For simplicity, we assume that $m_{\mathrm{RR}}^{0}$ and $p_{0}{ }^{0}$ are positive definite and $D=1$. To restore explicit contributions of $D$, one replaces the charges $\left(m_{\mathrm{RR}}, m_{\mathrm{RR}}^{0}, p_{0}, p_{0}{ }^{0}\right)$ to $\left(D m_{\mathrm{RR}}, D m_{\mathrm{RR}}^{0}, D p_{0}, D p_{0}{ }^{0}\right)$.

Following the discussion in (2.9), the supersymmetry condition is described by one differential and one algebraic equations with respect to $\mathcal{W}^{\mathrm{RR}}$ and $\mathcal{W}^{\mathcal{Q}}$ :

$$
\begin{aligned}
\left.D_{t} \mathcal{W}\right|_{*} & =0 & \rightarrow & \left.D_{t} \mathcal{W}^{\mathrm{RR}}\right|_{*} & =-\left.U_{*} D_{t} \mathcal{W}^{\mathcal{Q}}\right|_{*}, \\
\left.D_{U} \mathcal{W}\right|_{*} & =0 & \rightarrow & \mathcal{W}_{*}^{\mathrm{RR}} & =-\operatorname{Re} U_{*} \mathcal{W}_{*}^{\mathcal{Q}}
\end{aligned}
$$

It is useful to consider discriminants ${ }^{1}$ of the Ramond-Ramond flux superpotential $\mathcal{W}^{\mathrm{RR}}$ and of its derivative $\partial_{t} \mathcal{W}^{\mathrm{RR}}$ :

$$
\begin{aligned}
\Delta\left(\mathcal{W}^{\mathrm{RR}}\right) \equiv \Delta^{\mathrm{RR}}= & -27\left(m_{\mathrm{RR}}^{0} e_{\mathrm{RR} 0}\right)^{2}-54 m_{\mathrm{RR}}^{0} e_{\mathrm{RR} 0} m_{\mathrm{RR}} e_{\mathrm{RR}}+9\left(m_{\mathrm{RR}} e_{\mathrm{RR}}\right)^{2} \\
& +108\left(m_{\mathrm{RR}}\right)^{3} e_{\mathrm{RR} 0}-4 m_{\mathrm{RR}}^{0}\left(e_{\mathrm{RR}}\right)^{3} \\
\Delta\left(\partial_{t} \mathcal{W}^{\mathrm{RR}}\right) \equiv \lambda^{\mathrm{RR}}= & 12\left(3\left(m_{\mathrm{RR}}\right)^{2}-m_{\mathrm{RR}}^{0} e_{\mathrm{RR}}\right) .
\end{aligned}
$$

It is also useful to discuss discriminants of the (non)geometric flux superpotential $\mathcal{W}^{\mathcal{Q}}$ and of its derivative $\partial_{t} \mathcal{W}^{\mathcal{Q}}$ :

$$
\begin{aligned}
\Delta\left(\mathcal{W}^{\mathcal{Q}}\right) & \equiv \Delta^{\mathcal{Q}}=-27\left(p_{0}{ }^{0} e_{00}\right)^{2}-54 p_{0}{ }^{0} e_{00} p_{0} e_{0}+9\left(p_{0} e_{0}\right)^{2}-108\left(p_{0}\right)^{3} e_{00}+4 p_{0}{ }^{0}\left(e_{0}\right)^{3} \\
\Delta\left(\partial_{t} \mathcal{W}^{\mathcal{Q}}\right) & \equiv \lambda^{\mathcal{Q}}=12\left(3\left(p_{0}\right)^{2}+p_{0}{ }^{0} e_{0}\right) .
\end{aligned}
$$

Our strategy is as follows: First we investigate zeros of the Ramond-Ramond flux superpotential $\mathcal{W}^{\mathrm{RR}}$ and those of its covariant derivative $D_{t} \mathcal{W}^{\mathrm{RR}}$ by using the discriminants $\Delta^{\mathrm{RR}}$ and $\lambda^{\mathrm{RR}}$. Second we analyze the (non)geometric flux superpotential $\mathcal{W}^{\mathcal{Q}}$ in terms of the discriminants $\Delta^{\mathcal{Q}}$ and $\lambda^{\mathcal{Q}}$ in a parallel way. Third we evaluate possible supersymmetric vacua following the equations (3.4).

\footnotetext{
${ }^{1}$ The author would like to thank Tohru Eguchi for his introducing an essential idea of the usage of discriminants.
} 


\subsection{Ramond-Ramond flux superpotential}

\subsubsection{Solutions of $D_{t} \mathcal{W}^{\mathrm{RR}}=0$}

We formally describe a solution of $D_{t} \mathcal{W}^{\mathrm{RR}}=0$ :

$$
t_{*} \equiv t_{1 *}+i t_{2 *}=\frac{6\left(3 m_{\mathrm{RR}}^{0} e_{\mathrm{RR} 0}+m_{\mathrm{RR}} e_{\mathrm{RR}}\right)}{\lambda^{\mathrm{RR}}} \pm \frac{2 i \sqrt{3 \Delta^{\mathrm{RR}}}}{\lambda^{\mathrm{RR}}}
$$

The superpotential at this point is given by

$$
\mathcal{W}_{*}^{\mathrm{RR}}=-\frac{24 \Delta^{\mathrm{RR}}}{\left(\lambda^{\mathrm{RR}}\right)^{3}}\left(36\left(m_{\mathrm{RR}}\right)^{3}+36\left(m_{\mathrm{RR}}^{0}\right)^{2} e_{\mathrm{RR} 0}-3 m_{\mathrm{RR}} \lambda^{\mathrm{RR}}-4 i \operatorname{sign}\left(\lambda^{\mathrm{RR}}\right) m_{\mathrm{RR}}^{0} \sqrt{3 \Delta^{\mathrm{RR}}}\right) .
$$

These expressions are quite sensitive to signs of the discriminants $\Delta^{\mathrm{RR}}$ and $\lambda^{\mathrm{RR}}$.

If $\Delta^{\mathrm{RR}}$ is positive, $\lambda^{\mathrm{RR}}$ is always positive. Under this condition we find that the expression $t_{*}$ (3.7a) becomes a consistent solution and that the superpotential does not vanish:

$$
\begin{aligned}
t_{*} & =\frac{6\left(3 m_{\mathrm{RR}}^{0} e_{\mathrm{RR} 0}+m_{\mathrm{RR}} e_{\mathrm{RR}}\right)}{\lambda^{\mathrm{RR}}}-\frac{2 i \sqrt{3 \Delta^{\mathrm{RR}}}}{\lambda^{\mathrm{RR}}}, \\
\mathcal{W}_{*}^{\mathrm{RR}} & =-\frac{24 \Delta^{\mathrm{RR}}}{\left(\lambda^{\mathrm{RR}}\right)^{3}}\left(36\left(m_{\mathrm{RR}}\right)^{3}+36\left(m_{\mathrm{RR}}^{0}\right)^{2} e_{\mathrm{RR} 0}-3 m_{\mathrm{RR}} \lambda^{\mathrm{RR}}-4 i m_{\mathrm{RR}}^{0} \sqrt{3 \Delta^{\mathrm{RR}}}\right) .
\end{aligned}
$$

Here we chose the minus sign in front of $t_{2 *}$ in order that the Kähler potential $K_{+}=-\log \left[-i\left(t_{*}-\bar{t}_{*}\right)^{3}\right]$ is well-defined.

If $\Delta^{\mathrm{RR}}$ vanishes, $\lambda^{\mathrm{RR}}$ is non-negative. However, if $\lambda^{\mathrm{RR}}$ also vanishes, $t_{*}$ and $\mathcal{W}_{*}^{\mathrm{RR}}$ become singular. This is forbidden. In the case of positive $\lambda^{\mathrm{RR}}, t_{*}$ is real and $\mathcal{W}_{*}^{\mathrm{RR}}$ vanishes. Although this point is harmless as far as the equation $D_{t} \mathcal{W}^{\mathrm{RR}}=0$ is concerned, it should not be chosen as an admissible supersymmetric solution, because the metric and the curvature tensor become singular:

$$
K_{t \bar{t}}=-\frac{3}{(t-\bar{t})^{2}}, \quad R_{t t \bar{t}}^{t}=\frac{2}{(t-\bar{t})^{2}} .
$$

We conclude that if the discriminant $\Delta^{\mathrm{RR}}$ vanishes, there are no physical solutions.

If $\Delta^{\mathrm{RR}}$ is negative, $t_{2 *}$ in $(3.7 \mathrm{a})$ is ill-defined. This implies that there are no consistent solutions of the equation $\left.D_{t} \mathcal{W}^{\mathrm{RR}}\right|_{*}=0$, even though $\lambda^{\mathrm{RR}}$ is not restricted.

\subsubsection{Solutions of $\mathcal{W}^{\mathrm{RR}}=0$}

Here we look for a consistent solution which satisfies the equation $\mathcal{W}_{*}^{\mathrm{RR}}=0$. In this consideration it is also useful to classify physical solutions in terms of the discriminant $\Delta^{\mathrm{RR}}(3.5)$.

If $\Delta^{\mathrm{RR}}$ is positive, there are three distinct real roots $\left(e_{1}, e_{2}, e_{3}\right)$ of the equation $\mathcal{W}^{\mathrm{RR}}=0$. The superpotential and its Kähler covariant derivative are rewritten as

$$
\mathcal{W}^{\mathrm{RR}}=m_{\mathrm{RR}}^{0}\left(t-e_{1}\right)\left(t-e_{2}\right)\left(t-e_{3}\right), \quad e_{1}, e_{2}, e_{3} \in \mathbb{R},
$$




$$
D_{t} \mathcal{W}^{\mathrm{RR}}=-\frac{\mathcal{W}^{\mathrm{RR}}}{t-\bar{t}}\left(\frac{\bar{t}-e_{1}}{t-e_{1}}+\frac{\bar{t}-e_{2}}{t-e_{2}}+\frac{\bar{t}-e_{3}}{t-e_{3}}\right)
$$

The three real roots $e_{i}$ are related to the Ramond-Ramond flux charges:

$$
3 m_{\mathrm{RR}}=m_{\mathrm{RR}}^{0}\left(e_{1}+e_{2}+e_{3}\right), \quad e_{\mathrm{RR}}=m_{\mathrm{RR}}^{0}\left(e_{1} e_{2}+e_{2} e_{3}+e_{3} e_{1}\right), \quad e_{\mathrm{RR} 0}=-m_{\mathrm{RR}}^{0} e_{1} e_{2} e_{3} .
$$

We find a non-zero value of the covariant derivative at the points $t_{*}=e_{i}$. For instance, the value at $t_{*}=e_{1}$ is

$$
\left.D_{t} \mathcal{W}^{\mathrm{RR}}\right|_{t_{*}=e_{1}}=-3 m_{\mathrm{RR}}^{0}\left(e_{1}-e_{2}\right)\left(e_{1}-e_{3}\right) \neq 0 \text {. }
$$

This value itself is finite. However, the Kähler metric and the curvature (3.9) become singular. Then we cannot choose this solution as an attractor point. The other two zeros $e_{2}$ and $e_{3}$ give the same situations. Thus there are no finite solutions of $\mathcal{W}^{\mathrm{RR}}=0$ if $\Delta^{\mathrm{RR}}$ is positive.

If $\Delta^{\mathrm{RR}}$ vanishes, $\lambda^{\mathrm{RR}}$ is non-negative. When $\lambda^{\mathrm{RR}}$ is positive, the equation $\mathcal{W}^{\mathrm{RR}}=0$ has two coincident real roots $e_{1}$ and a distinct real root $e_{2}$. When $\lambda^{\mathrm{RR}}$ vanishes, the three roots coincide with each other. In both cases the superpotential and its covariant derivative are

$$
\begin{aligned}
\mathcal{W}^{\mathrm{RR}} & =m_{\mathrm{RR}}^{0}\left(t-e_{1}\right)^{2}\left(t-e_{2}\right), \quad e_{1}, e_{2} \in \mathbb{R}, \\
D_{t} \mathcal{W}^{\mathrm{RR}} & =-\frac{\mathcal{W}^{\mathrm{RR}}}{t-\bar{t}}\left(\frac{2\left(\bar{t}-e_{1}\right)}{t-e_{1}}+\frac{\bar{t}-e_{2}}{t-e_{2}}\right) .
\end{aligned}
$$

The relations among the flux charges and the roots are

$$
3 m_{\mathrm{RR}}=m_{\mathrm{RR}}^{0}\left(2 e_{1}+e_{2}\right), \quad e_{\mathrm{RR}}=m_{\mathrm{RR}}^{0}\left(\left(e_{1}\right)^{2}+2 e_{1} e_{2}\right), \quad e_{\mathrm{RR} 0}=-m_{\mathrm{RR}}^{0}\left(e_{1}\right)^{2} e_{2} .
$$

We find that the covariant derivatives of the superpotential vanish at the points $t_{*}=e_{i}$ :

$$
\left.D_{t} \mathcal{W}^{\mathrm{RR}}\right|_{t_{*}=e_{1}}=0,\left.\quad D_{t} \mathcal{W}^{\mathrm{RR}}\right|_{t_{*}=e_{2}}=0
$$

These values are finite. However, the Kähler metric and the curvature (3.9) become singular in the same reason as in $\Delta^{\mathrm{RR}}>0$. They are inadmissible to physical solutions.

If $\Delta^{\mathrm{RR}}$ is negative, the equation $\mathcal{W}^{\mathrm{RR}}=0$ has one real root $e_{1}$ and a pair of complex roots $(\alpha, \bar{\alpha})$. Then the superpotential and its covariant derivative are rewritten as

$$
\begin{aligned}
\mathcal{W}^{\mathrm{RR}} & =m_{\mathrm{RR}}^{0}\left(t-e_{1}\right)(t-\alpha)(t-\bar{\alpha}), \quad e_{1} \in \mathbb{R}, \quad \alpha \in \mathbb{C}, \\
D_{t} \mathcal{W}^{\mathrm{RR}} & =-\frac{\mathcal{W}^{\mathrm{RR}}}{t-\bar{t}}\left(\frac{\bar{t}-e_{1}}{t-e_{1}}+\frac{\bar{t}-\alpha}{t-\alpha}+\frac{\bar{t}-\bar{\alpha}}{t-\bar{\alpha}}\right) .
\end{aligned}
$$

The three roots are related to the flux charges:

$$
3 m_{\mathrm{RR}}=m_{\mathrm{RR}}^{0}\left(e_{1}+\alpha+\bar{\alpha}\right), \quad e_{\mathrm{RR}}=m_{\mathrm{RR}}^{0}\left(e_{1}(\alpha+\bar{\alpha})+|\alpha|^{2}\right), \quad e_{\mathrm{RR} 0}=-m_{\mathrm{RR}}^{0} e_{1}|\alpha|^{2} .
$$


The solutions are explicitly given by

$$
\begin{aligned}
e_{1} & =-\frac{1}{m_{\mathrm{RR}}^{0}}\left(-3 m_{\mathrm{RR}}+2 m_{\mathrm{RR}}^{0}(\mathrm{Re} \alpha)\right) \\
(\mathrm{Re} \alpha) & =\frac{\lambda^{\mathrm{RR}}+\left(F_{\mathrm{RR}}\right)^{2 / 3}+12 m_{\mathrm{RR}}\left(F_{\mathrm{RR}}\right)^{1 / 3}}{12 m_{\mathrm{RR}}^{0}\left(F_{\mathrm{RR}}\right)^{1 / 3}} \quad\left(\text { if } F_{\mathrm{RR}}>0\right) \\
\text { or } \quad(\operatorname{Re} \alpha) & =-\frac{1}{24 m_{\mathrm{RR}}^{0}\left(F_{\mathrm{RR}}\right)^{1 / 3}}\left(\left(\lambda^{\mathrm{RR}}+\left(F_{\mathrm{RR}}\right)^{2 / 3}\right) \pm \sqrt{3} i\left(\lambda^{\mathrm{RR}}-\left(F_{\mathrm{RR}}\right)^{2 / 3}\right)-24 m_{\mathrm{RR}}\left(F_{\mathrm{RR}}\right)^{1 / 3}\right) \\
& =\frac{\lambda^{\mathrm{RR}}+\left(G_{\mathrm{RR}}\right)^{2 / 3}+12 m_{\mathrm{RR}}\left(G_{\mathrm{RR}}\right)^{1 / 3}}{12 m_{\mathrm{RR}}^{0}\left(G_{\mathrm{RR}}\right)^{1 / 3}} \quad\left(\text { if } F_{\mathrm{RR}}=-G_{\mathrm{RR}}<0\right) \\
(\operatorname{Im} \alpha)^{2} & =\frac{1}{m_{\mathrm{RR}}^{0}\left(e_{\mathrm{RR}}-6 m_{\mathrm{RR}}(\operatorname{Re} \alpha)+3 m_{\mathrm{RR}}^{0}(\operatorname{Re} \alpha)^{2}\right)} \\
F_{\mathrm{RR}} & =108\left(m_{\mathrm{RR}}^{0}\right)^{2} e_{\mathrm{RR} 0}+12 m_{\mathrm{RR}}^{0} \sqrt{-3 \Delta^{\mathrm{RR}}}+108\left(m_{\mathrm{RR}}\right)^{3}-9 \lambda^{\mathrm{RR}} m_{\mathrm{RR}}
\end{aligned}
$$

Note that $F_{\mathrm{RR}}$ cannot vanish otherwise $t_{*}=\alpha$ goes to infinity. In order that the above expressions provide a solution of $\mathcal{W}_{*}^{\mathrm{RR}}=0$ and $\left.D_{t} \mathcal{W}^{\mathrm{RR}}\right|_{*} \neq 0$, the square of the imaginary part of $\alpha$ has to be positive definite:

$$
3 m_{\mathrm{RR}}^{0}(\operatorname{Re} \alpha)^{2}-6 m_{\mathrm{RR}}(\operatorname{Re} \alpha)+e_{\mathrm{RR}}>0 .
$$

The discriminant of the function of $(\operatorname{Re} \alpha)$ in the left-hand side is nothing but $\lambda^{\mathrm{RR}}$. If this is nonnegative, there exist the following points where $(\operatorname{Im} \alpha)$ vanishes:

$$
(\operatorname{Re} \alpha)=\frac{1}{6 m_{\mathrm{RR}}^{0}}\left(6 m_{\mathrm{RR}} \pm \sqrt{\lambda^{\mathrm{RR}}}\right) .
$$

However, this is inconsistent with $\Delta^{\mathrm{RR}}<0$ that gives one real and a pair of complex zeros. Then we find that $\lambda^{\mathrm{RR}}<0$ is necessary to obtain a solution of $\mathcal{W}_{*}^{\mathrm{RR}}=0$ with $\left.D_{t} \mathcal{W}^{\mathrm{RR}}\right|_{*} \neq 0$. Since the root $t_{*}=e_{1}$ gives singular curvature, the consistent solution is only given by $t_{*}=\alpha$.

\section{3 (Non)geometric flux superpotential}

In this subsection we investigate features of the (non)geometric flux superpotential. Since the function $\mathcal{W}^{\mathcal{Q}}$ is similar to $\mathcal{W}^{\mathrm{RR}}$, we can evaluate this sector in a parallel way as in the previous subsection. First we look for a solution of $D_{t} \mathcal{W}^{\mathcal{Q}}=0$. Next we analyze a condition $\mathcal{W}^{\mathcal{Q}}=0$ by using the discriminants $\Delta^{\mathcal{Q}}$ and $\lambda^{\mathcal{Q}}$ in (3.6).

\subsubsection{Solutions of $D_{t} \mathcal{W}^{\mathcal{Q}}=0$}

Let us investigate consistent conditions to satisfy the equation $D_{t} \mathcal{W}^{\mathcal{Q}}=0$. We formally describe a solution of $D_{t} \mathcal{W}^{\mathcal{Q}}=0$ as follows:

$$
t_{*} \equiv t_{1 *}+i t_{2 *}=-\frac{6\left(3 p_{0}^{0} e_{00}+p_{0} e_{0}\right)}{\lambda^{\mathcal{Q}}} \pm \frac{2 i \sqrt{3 \Delta^{\mathcal{Q}}}}{\lambda^{\mathcal{Q}}}
$$


The superpotential at this point is given by

$$
\mathcal{W}_{*}^{\mathcal{Q}}=-\frac{24 \Delta^{\mathcal{Q}}}{\left(\lambda^{\mathcal{Q}}\right)^{3}}\left(36\left(p_{0}\right)^{3}-36\left(p_{0}{ }^{0}\right)^{2} e_{00}-3 p_{0} \lambda^{\mathcal{Q}}-4 i \operatorname{sign}\left(\lambda^{\mathcal{Q}}\right) p_{0}{ }^{0} \sqrt{3 \Delta^{\mathcal{Q}}}\right) .
$$

Consistency of the above formal expression is evaluated in terms of the discriminants $\Delta^{\mathcal{Q}}$ and $\lambda^{\mathcal{Q}}$ as in the previous subsection.

If $\Delta^{\mathcal{Q}}$ is positive, $\lambda^{\mathcal{Q}}$ is always positive. Under this condition we find that $t_{*}(3.17 \mathrm{a})$ becomes a consistent solution with non-vanishing superpotential:

$$
\begin{aligned}
t_{*} & =-\frac{6\left(3 p_{0}{ }^{0} e_{00}+p_{0} e_{0}\right)}{\lambda^{\mathcal{Q}}}-\frac{2 i \sqrt{3 \Delta^{\mathcal{Q}}}}{\lambda^{\mathcal{Q}}}, \\
\mathcal{W}_{*}^{\mathcal{Q}} & =-\frac{24 \Delta^{\mathcal{Q}}}{\left(\lambda^{\mathcal{Q}}\right)^{3}}\left(36\left(p_{0}\right)^{3}-36\left(p_{0}^{0}\right)^{2} e_{00}-3 p_{0} \lambda^{\mathcal{Q}}-4 i p_{0}{ }^{0} \sqrt{3 \Delta^{\mathcal{Q}}}\right) .
\end{aligned}
$$

Here we have already chose the negative sign in front of $t_{2 *}$ to realize a well-defined Kähler potential. We find the Kähler metric is non-degenerated and the curvature is finite.

If $\Delta^{\mathcal{Q}}$ vanishes, $\lambda^{\mathcal{Q}}$ is non-negative. However if $\lambda^{\mathcal{Q}}$ is zero, $t_{1 *}$ in $(3.17 \mathrm{a})$ and $\mathcal{W}_{*}^{\mathcal{Q}}(3.17 \mathrm{~b})$ are ill-defined. Then only the positive $\lambda^{\mathcal{Q}}$ is allowed. In this case, $t_{*}$ is reduced to a real value and $\mathcal{W}_{*}^{\mathcal{Q}}$ vanishes. It cannot be chosen as a physical solution to realize a well-defined supersymmetric solution, because the curvature tensor (3.9) goes to infinity. We conclude that there are no admissible solutions of $D_{t} \mathcal{W}^{\mathcal{Q}}=0$ if $\Delta^{\mathcal{Q}}$ vanishes.

If $\Delta^{\mathcal{Q}}$ is negative, the expression $t_{2 *}$ in (3.17a) becomes ill-defined. This implies that there are no consistent solutions of the equation $\left.D_{t} \mathcal{W}^{\mathcal{Q}}\right|_{*}=0$, even though the discriminant $\lambda^{\mathcal{Q}}$ is not restricted.

\subsubsection{Solutions of $\mathcal{W}^{\mathcal{Q}}=0$}

Here we look for a consistent solution of the equation $\mathcal{W}_{*}^{\mathcal{Q}}=0$. If $\Delta^{\mathcal{Q}}$ is positive or zero, there are no consistent solutions to realize supersymmetric vacua as in the previous subsection. Then we focus on the case of the negative valued $\Delta^{\mathcal{Q}}$. In this case, the equation $\mathcal{W}^{\mathcal{Q}}=0$ has one real root $e_{1}$ and a pair of complex roots $(\alpha, \bar{\alpha})$. The superpotential and its covariant derivative are written as

$$
\begin{aligned}
\mathcal{W}^{\mathcal{Q}} & =p_{0}^{0}\left(t-e_{1}\right)(t-\alpha)(t-\bar{\alpha}), \quad e_{1} \in \mathbb{R}, \quad \alpha \in \mathbb{C}, \\
D_{t} \mathcal{W}^{\mathcal{Q}} & =-\frac{\mathcal{W}^{\mathcal{Q}}}{t-\bar{t}}\left(\frac{\bar{t}-e_{1}}{t-e_{1}}+\frac{\bar{t}-\alpha}{t-\alpha}+\frac{\bar{t}-\bar{\alpha}}{t-\bar{\alpha}}\right) .
\end{aligned}
$$

The three roots are related to the flux charges:

$$
3 p_{0}=p_{0}^{0}\left(e_{1}+\alpha+\bar{\alpha}\right), \quad e_{0}=-p_{0}^{0}\left(e_{1}(\alpha+\bar{\alpha})+|\alpha|^{2}\right), \quad e_{00}=p_{0}^{0} e_{1}|\alpha|^{2} .
$$

The solutions are given by

$$
e_{1}=-\frac{1}{p_{0} 0}\left(-3 p_{0}+2 p_{0}^{0}(\operatorname{Re} \alpha)\right)
$$




$$
\begin{aligned}
(\operatorname{Re} \alpha) & =\frac{\lambda^{\mathcal{Q}}+\left(F_{\mathcal{Q}}\right)^{2 / 3}+12 p_{0}\left(F_{\mathcal{Q}}\right)^{1 / 3}}{12 p_{0}^{0}\left(F_{\mathcal{Q}}\right)^{1 / 3}} \quad\left(\text { if } F_{\mathcal{Q}}>0\right) \\
\text { or } \quad(\operatorname{Re} \alpha) & =-\frac{1}{24 p_{0}^{0}\left(F_{\mathcal{Q}}\right)^{1 / 3}}\left(\left(\lambda^{\mathcal{Q}}+\left(F_{\mathcal{Q}}\right)^{2 / 3}\right) \pm \sqrt{3} i\left(\lambda^{\mathcal{Q}}-\left(F_{\mathcal{Q}}\right)^{2 / 3}\right)-24 p_{0}\left(F_{\mathcal{Q}}\right)^{1 / 3}\right) \\
& =\frac{\lambda^{\mathcal{Q}}+\left(G_{\mathcal{Q}}\right)^{2 / 3}+12 p_{0}\left(G_{\mathcal{Q}}\right)^{1 / 3}}{12 p_{0}^{0}\left(G_{\mathcal{Q}}\right)^{1 / 3}} \quad\left(\text { if } F_{\mathcal{Q}}=-G_{\mathcal{Q}}<0\right) \\
(\operatorname{Im} \alpha)^{2} & =\frac{1}{p_{0}^{0}}\left(-e_{0}-6 p_{0}(\operatorname{Re} \alpha)+3 p_{0}^{0}(\operatorname{Re} \alpha)^{2}\right) \\
F_{\mathcal{Q}} & =-108\left(p_{0}{ }^{0}\right)^{2} e_{00}+12 p_{0}{ }^{0} \sqrt{-3 \Delta^{\mathcal{Q}}}+108\left(p_{0}\right)^{3}-9 \lambda^{\mathcal{Q}} p_{0}
\end{aligned}
$$

Note that $F_{\mathcal{Q}}$ is non-zero otherwise $t_{*}=\alpha$ goes to infinity. Since we have already assumed $p_{0}{ }^{0}>0$, the following inequality should be imposed:

$$
3 p_{0}^{0}(\operatorname{Re} \alpha)^{2}-6 p_{0}(\operatorname{Re} \alpha)-e_{0}>0 .
$$

The discriminant of the function of $(\operatorname{Re} \alpha)$ in the left-hand side is nothing but $\lambda^{\mathcal{Q}}$. If this is nonnegative, there exist the following points where $(\operatorname{Im} \alpha)$ vanishes:

$$
(\operatorname{Re} \alpha)=\frac{1}{6 p_{0} 0}\left(6 p_{0} \pm \sqrt{\lambda^{\mathcal{Q}}}\right) .
$$

However, this is inconsistent with the condition $\Delta^{\mathcal{Q}}<0$ which gives one real and a pair of complex zeros. Then $\lambda^{\mathcal{Q}}<0$ is necessary to obtain a solution of $\mathcal{W}_{*}^{\mathcal{Q}}=0$ with $\left.D_{t} \mathcal{W}^{\mathcal{Q}}\right|_{*} \neq 0$. Since $t_{*}=e_{1}$ gives ill-defined curvature, the consistent solution is only given by $t_{*}=\alpha$.

\subsection{Supersymmetric vacua}

We have already studied various situations when the superpotentials $\mathcal{W}^{\mathrm{RR}}$ and $\mathcal{W}^{\mathcal{Q}}$ and/or their covariant derivatives $D_{t} \mathcal{W}^{\mathrm{RR}}$ and $D_{t} \mathcal{W}^{\mathcal{Q}}$ have zeros. The signs of the discriminants of the superpotentials characterize admissible solutions. Here we classify supersymmetric flux attractor vacua which satisfy (3.4).

Consider the case that both the two discriminants $\Delta^{\mathrm{RR}}$ and $\Delta^{\mathcal{Q}}$ are positive. There exists a solution which satisfies $D_{t} \mathcal{W}^{\mathrm{RR}}=0, D_{t} \mathcal{W}^{\mathcal{Q}}=0, \mathcal{W}^{\mathrm{RR}} \neq 0$ and $\mathcal{W}^{\mathcal{Q}} \neq 0$. Here obtain the following equations from (3.8) and (3.18):

$$
\begin{aligned}
\left.D_{t} \mathcal{W}\right|_{*} & =\left.D_{t} \mathcal{W}^{\mathrm{RR}}\right|_{*}+\left.U_{*} D_{t} \mathcal{W}^{\mathcal{Q}}\right|_{*}=0,\left.\quad D_{t} \mathcal{W}^{\mathrm{RR}}\right|_{*}=\left.D_{t} \mathcal{W}^{\mathcal{Q}}\right|_{*}=0, \\
\left.D_{U} \mathcal{W}\right|_{*} & =\frac{1}{\operatorname{Im} U}\left(\mathcal{W}_{*}^{\mathrm{RR}}+\operatorname{Re} U_{*} \mathcal{W}_{*}^{\mathcal{Q}}\right)=0 \\
\mathcal{W}_{*} & =\mathcal{W}_{*}^{\mathrm{RR}}+U_{*} \mathcal{W}_{*}^{\mathcal{Q}}=i \operatorname{Im} U_{*} \mathcal{W}_{*}^{\mathcal{Q}} \\
t_{*}^{\mathrm{RR}} & =\frac{6\left(3 m_{\mathrm{RR}}^{0} e_{\mathrm{RR} 0}+m_{\mathrm{RR}} e_{\mathrm{RR}}\right)}{\lambda^{\mathrm{RR}}}-\frac{2 i \sqrt{3 \Delta^{\mathrm{RR}}}}{\lambda^{\mathrm{RR}}}
\end{aligned}
$$




$$
\begin{aligned}
t_{*}^{\mathcal{Q}} & =-\frac{6\left(3 p_{0}^{0} e_{00}+p_{0} e_{0}\right)}{\lambda^{\mathcal{Q}}}-\frac{2 i \sqrt{3 \Delta^{\mathcal{Q}}}}{\lambda^{\mathcal{Q}}}, \\
\mathcal{W}_{*}^{\mathrm{RR}} & =-\frac{24 \Delta^{\mathrm{RR}}}{\left(\lambda^{\mathrm{RR}}\right)^{3}}\left(36\left(m_{\mathrm{RR}}\right)^{3}+36\left(m_{\mathrm{RR}}^{0}\right)^{2} e_{\mathrm{RR} 0}-3 m_{\mathrm{RR}} \lambda^{\mathrm{RR}}-4 i m_{\mathrm{RR}}^{0} \sqrt{3 \Delta^{\mathrm{RR}}}\right), \\
\mathcal{W}_{*}^{\mathcal{Q}} & =-\frac{24 \Delta^{\mathcal{Q}}}{\left(\lambda^{\mathcal{Q}}\right)^{3}}\left(36\left(p_{0}\right)^{3}-36\left(p_{0}^{0}\right)^{2} e_{00}-3 p_{0} \lambda^{\mathcal{Q}}-4 i p_{0}{ }^{0} \sqrt{3 \Delta^{\mathcal{Q}}}\right) .
\end{aligned}
$$

Since the two solutions $t_{*}^{\mathrm{RR}}$ and $t_{*}^{\mathcal{Q}}$ have to coincide with each other, we find a non-trivial relation:

$$
\frac{3 m_{\mathrm{RR}}^{0} e_{\mathrm{RR} 0}+m_{\mathrm{RR}} e_{\mathrm{RR}}}{\lambda^{\mathrm{RR}}}=-\frac{3 p_{0}^{0} e_{00}+p_{0} e_{0}}{\lambda^{\mathcal{Q}}}, \quad \frac{\sqrt{\Delta^{\mathrm{RR}}}}{\lambda^{\mathrm{RR}}}=\frac{\sqrt{\Delta^{\mathcal{Q}}}}{\lambda^{\mathcal{Q}}} .
$$

We can fix only the real part of the variable $U$ by

$$
\operatorname{Re} U_{*}=-\frac{\mathcal{W}_{*}^{\mathrm{RR}}}{\mathcal{W}_{*}^{\mathcal{Q}}}
$$

whilst the imaginary part remains unfixed. This indicates that the dilaton $(2.1 \mathrm{~d})$ is not fixed. The value of the superpotential $\mathcal{W}_{*}$ also contains $\operatorname{Im} U$. However, this does not explicitly appear in the cosmological constant $\Lambda=-3 \mathrm{e}^{K}\left|\mathcal{W}_{*}\right|^{2}$ :

$$
-3 \mathrm{e}^{K}\left|\mathcal{W}_{*}\right|^{2}=\frac{3}{2\left(t_{2}^{\mathcal{Q}}\right)^{3}} \frac{1}{\left[\operatorname{Re}\left(\mathcal{C} \mathcal{G}_{0}\right)\right]^{2}}\left|\mathcal{W}_{*}^{\mathcal{Q}}\right|^{2}=-\frac{4}{\left[\operatorname{Re}\left(\mathcal{C G}_{0}\right)\right]^{2}} \sqrt{\frac{\Delta^{\mathcal{Q}}}{3}}
$$

The value $\operatorname{Re}\left(\mathcal{C G}_{0}\right)$, which should be non-zero to realize a well-defined Kähler potential $(2.1 \mathrm{~b})$, is not fixed by the attractor equations, either. However this should be very large under the supergravity approximation: The exponent of the expectation value of the dilaton gives the sting coupling constant. This should be very small. This restriction imposes that the compensator $\mathcal{C}(\mathrm{C} .11 \mathrm{~b})$ is very large. Then the cosmological constant (3.27) becomes very small. This solution realizes a supersymmetric AdS vacuum. The stability of the system has already been guaranteed by [46] in a generic form, where all mass eigenvalues satisfy the Breitenlohner-Freedman criterion [47]. This result differs from that of [44] where only the Minkowski vacua is realized. This difference comes from the introduction of the Ramond-Ramond flux charges. We will come back to this issue in later sections.

Next, let us consider the case that both of $\Delta^{\mathrm{RR}}$ and $\Delta^{\mathcal{Q}}$ are negative. There exists another attractor point which satisfies $\mathcal{W}^{\mathrm{RR}}=0, \mathcal{W}^{\mathcal{Q}}=0, D_{t} \mathcal{W}^{\mathrm{RR}} \neq 0$ and $D_{t} \mathcal{W}^{\mathcal{Q}} \neq 0$. We can see a non-trivial relation between the Ramond-Ramond flux charges and the (non)geometric flux charges via the equations $\mathcal{W}^{\mathrm{RR}}=0$ and $\mathcal{W}^{\mathcal{Q}}=0$. The former gives a solution $t_{*}=\alpha^{\mathrm{RR}}\left(e_{\mathrm{RR} 0}, e_{\mathrm{RR}}, m_{\mathrm{RR}}, m_{\mathrm{RR}}^{0}\right)$ in (3.14), while the latter yields $t_{*}=\alpha^{\mathcal{Q}}\left(e_{00}, e_{0}, p_{0}, p_{0}{ }^{o}\right)$ in (3.21). These two solutions have to coincide with each other:

$$
\begin{aligned}
\alpha^{\mathrm{RR}} & =\alpha^{\mathcal{Q}} \\
\operatorname{Re} \alpha^{\mathrm{RR}} & =\frac{\lambda^{\mathrm{RR}}+\left(F_{\mathrm{RR}}\right)^{2 / 3}+12 m_{\mathrm{RR}}\left(F_{\mathrm{RR}}\right)^{1 / 3}}{12 m_{\mathrm{RR}}^{0}\left(F_{\mathrm{RR}}\right)^{1 / 3}}
\end{aligned}
$$




$$
\begin{aligned}
\operatorname{Re} \alpha^{\mathcal{Q}} & =\frac{\lambda^{\mathcal{Q}}+\left(F_{\mathcal{Q}}\right)^{2 / 3}+12 p_{0}\left(F_{\mathcal{Q}}\right)^{2 / 3}}{12 p_{0}^{0}\left(F_{\mathcal{Q}}\right)^{1 / 3}} \\
\left(\operatorname{Im} \alpha^{\mathrm{RR}}\right)^{2} & =\frac{1}{m_{\mathrm{RR}}^{0}}\left(e_{\mathrm{RR}}-6 m_{\mathrm{RR}}\left(\operatorname{Re} \alpha^{\mathrm{RR}}\right)+3 m_{\mathrm{RR}}^{0}\left(\operatorname{Re} \alpha^{\mathrm{RR}}\right)\right) \\
\left(\operatorname{Im} \alpha^{\mathcal{Q}}\right)^{2} & =\frac{1}{p_{0}^{0}}\left(-e_{0}-6 p_{0}\left(\operatorname{Re} \alpha^{\mathcal{Q}}\right)+3 p_{0}^{0}\left(\operatorname{Re} \alpha^{\mathcal{Q}}\right)^{2}\right) \\
e_{1}^{\mathrm{RR}} & =-\frac{1}{m_{\mathrm{RR}}^{0}}\left(-3 m_{\mathrm{RR}}+2 m_{\mathrm{RR}}^{0}\left(\operatorname{Re} \alpha^{\mathrm{RR}}\right)\right) \\
e_{1}^{\mathcal{Q}} & =-\frac{1}{p_{0}^{0}}\left(-3 p_{0}+2 p_{0}^{0}\left(\operatorname{Re} \alpha^{\mathcal{Q}}\right)\right) \\
F_{\mathrm{RR}} & =108\left(m_{\mathrm{RR}}^{0}\right)^{2} e_{\mathrm{RR} 0}+12 m_{\mathrm{RR}}^{0} \sqrt{-3 \Delta^{\mathrm{RR}}}+108\left(m_{\mathrm{RR}}\right)^{3}-9 \lambda^{\mathrm{RR}} m_{\mathrm{RR}} \\
F_{\mathcal{Q}} & =-108\left(p_{0}^{0}\right)^{2} e_{00}+12 p_{0}^{0} \sqrt{-3 \Delta^{\mathcal{Q}}}+108\left(p_{0}\right)^{3}-9 \lambda^{\mathcal{Q}} p_{0} .
\end{aligned}
$$

We can stabilize the variable $U$ in the following way:

$$
\begin{aligned}
U_{*} & =-\frac{\left.D_{t} \mathcal{W}^{\mathrm{RR}}\right|_{t_{*}=\alpha}}{\left.D_{t} \mathcal{W}^{\mathcal{Q}}\right|_{t_{*}=\alpha}} \\
\left.D_{t} \mathcal{W}^{\mathrm{RR}}\right|_{t_{*}=\alpha} & =-2 i m_{\mathrm{RR}}^{0}\left(\operatorname{Im} \alpha^{\mathrm{RR}}\right)\left[3\left(\frac{m_{\mathrm{RR}}}{m_{\mathrm{RR}}^{0}}-\left(\operatorname{Re} \alpha^{\mathrm{RR}}\right)\right)-i\left(\operatorname{Im} \alpha^{\mathrm{RR}}\right)\right], \\
\left.D_{t} \mathcal{W}^{\mathcal{Q}}\right|_{t_{*}=\alpha} & =-2 i p_{0}^{0}\left(\operatorname{Im} \alpha^{\mathcal{Q}}\right)\left[3\left(\frac{p_{0}}{p_{0}^{0}}-\left(\operatorname{Re} \alpha^{\mathcal{Q}}\right)\right)-i\left(\operatorname{Im} \alpha^{\mathcal{Q}}\right)\right]
\end{aligned}
$$

where we used $\operatorname{Im} U \neq 0$ because of finiteness of the curvature tensor $R^{U} U \bar{U}$. The vanishing superpotential sets the cosmological constant to be zero. Then a supersymmetric Minkowski vacuum is realized. This configuration is interpreted that the internal space $\mathcal{M}$ is reduced to a parallelizable twisted torus [12].

We discuss other situations: (i) There are no attractor solutions to satisfy the equations (3.4) if the relative signs of the two discriminants are different; i.e., $\Delta^{\mathrm{RR}} \cdot \Delta^{\mathcal{Q}}<0$. (ii) Apart from the attractor solutions where the moduli are stabilized, there exist non-attractor solutions which satisfy the supersymmetry condition (3.4). Due to the lack of the number of equations, however, the moduli $t$ and $U$ are not fixed at all. These solutions do not provide vanishing superpotentials. Then the vacua are realized as AdS spaces.

\section{Example 2: a model without Ramond-Ramond flux charges}

In this section we study a model without the Ramond-Ramond flux charges $e_{\mathrm{RR} \check{A}}=0=m_{\mathrm{RR}}^{\check{A}}$. The total superpotential $\mathcal{W}$ and its covariant derivatives are reduced to

$$
\begin{aligned}
\mathcal{W} & =U \mathcal{W}^{\mathcal{Q}}, \\
\mathcal{W}^{\mathcal{Q}} & =-e_{00}-e_{0 \check{a}} t^{\check{a}}-3 p_{0}{ }^{\check{c}} D_{\check{a} \check{b} \check{c}} t^{\check{a}} t^{\check{b}}+p_{0}{ }^{0} D_{\check{a} \check{b} \check{c} \check{c}} t^{\check{t}} t^{\check{b}} t^{\check{c}},
\end{aligned}
$$




$$
D_{\check{a}} \mathcal{W}=U D_{\check{a}} \mathcal{W}^{\mathcal{Q}}, \quad D_{U} \mathcal{W}=i \frac{\operatorname{Re} U}{\operatorname{Im} U} \mathcal{W}^{\mathcal{Q}}
$$

We imposed $\operatorname{Im} U \neq 0$. In supersymmetric solutions, the following equations have to be satisfied:

$$
\begin{aligned}
D_{\check{a}} \mathcal{W}=0 & \leftrightarrow & D_{\check{a}} \mathcal{W}^{\mathcal{Q}}=0, \\
D_{U} \mathcal{W}=0 & \leftrightarrow & \operatorname{Re} U \mathcal{W}^{\mathcal{Q}}=0 .
\end{aligned}
$$

In the single modulus model as in section 3.3, we obtain a solution (3.18) consistent with (4.2):

$$
\begin{aligned}
\Delta^{\mathcal{Q}} & =-27\left(p_{0}{ }^{0} e_{00}\right)^{2}-54 p_{0}{ }^{0} e_{00} p_{0} e_{0}+9\left(p_{0} e_{0}\right)^{2}-108\left(p_{0}\right)^{3} e_{00}+4 p_{0}{ }^{0}\left(e_{0}\right)^{3}>0 \\
\lambda^{\mathcal{Q}} & =12\left(3\left(p_{0}\right)^{2}+p_{0}{ }^{0} e_{0}\right)>0 \\
t_{*} & =-\frac{6\left(3 p_{0}{ }^{0} e_{00}+p_{0} e_{0}\right)}{\lambda^{\mathcal{Q}}}-\frac{2 i \sqrt{3 \Delta^{\mathcal{Q}}}}{\lambda^{\mathcal{Q}}} \\
\mathcal{W}_{*}^{\mathcal{Q}} & =-\frac{24 \Delta^{\mathcal{Q}}}{\left(\lambda^{\mathcal{Q}}\right)^{3}}\left(36\left(p_{0}\right)^{3}-36\left(p_{0}^{0}\right)^{2} e_{00}-3 p_{0} \lambda^{\mathcal{Q}}-4 i p_{0}{ }^{0} \sqrt{3 \Delta^{\mathcal{Q}}}\right) \\
\operatorname{Re} U_{*} & =0
\end{aligned}
$$

Here we chose that $t_{2 *}$ is negative in order that the Kähler potential is well-defined. The scalar potential at this point is described as

$$
V_{*}=-\frac{4}{\left[\operatorname{Re}\left(\mathcal{C G}_{0}\right)\right]^{2}} \sqrt{\frac{\Delta^{\mathcal{Q}}}{3}} .
$$

In this model the attractor equations (4.2) can fix only the real part of the variable $U$, while its imaginary part is kept unfixed. Due to this, the value $\operatorname{Re}\left(\mathcal{C G}_{0}\right)$ is unfixed. However, this should be very large under the supergravity approximation. The only one condition is that $\operatorname{Re}\left(\mathcal{C G}_{0}\right)$ does not vanish in order to realize a well-defined Kähler potential (2.1b). This result again differs from that of [44]. There would be at least two possibilities: (i) The prepotential $\mathcal{F}$ in (2.5) would not be an appropriate form to find a Minkowski vacuum. (ii) The attractor equations [44] based on the work [41] might not be the most generic equations to find all flux vacua. It would be interesting to fill gaps between our result and that of [44].

\section{Example 3: models on $S U(3)$-structure manifold without Ramond- Ramond flux charges}

Here let us analyze a model compactified without the Ramond-Ramond flux charges and nongeometric flux charges. In this model we set

$$
e_{\mathrm{RR} \check{A}}=0, \quad m_{\mathrm{RR}}^{\check{A}}=0, \quad p_{0}^{\check{A}}=0, \quad q^{0 \check{A}}=0 .
$$


The total superpotential $\mathcal{W}$ and its covariant derivatives are reduced to

$$
\begin{aligned}
\mathcal{W} & =U \mathcal{W}^{\mathcal{Q}}, \\
\mathcal{W}^{\mathcal{Q}} & =-e_{00}-e_{0 \check{a}} t^{\check{a}}, \\
D_{\breve{a}} \mathcal{W} & =U D_{\check{a}} \mathcal{W}^{\mathcal{Q}}, \quad D_{U} \mathcal{W}=i \frac{\operatorname{Re} U}{\operatorname{Im} U} \mathcal{W}^{\mathcal{Q}}, \\
D_{\check{b}} D_{\check{a}} \mathcal{W} & =i U C_{\check{a} \check{b} \check{c}}\left(K_{+}\right)^{\check{c} \breve{d}} D_{\breve{d}} \overline{\mathcal{W}} \mathcal{\mathcal { Q }} \\
D_{U} D_{\breve{a}} \mathcal{W} & =i \frac{\operatorname{Re} U}{\operatorname{Im} U} D_{\breve{a}} \mathcal{W}^{\mathcal{Q}}, \quad D_{U} D_{U} \mathcal{W}=-\frac{U+2 \bar{U}}{2(\operatorname{Im} U)^{2}} \mathcal{W}^{\mathcal{Q}} .
\end{aligned}
$$

Let us first consider the case that $D_{P} \mathcal{W}=0$ is satisfied. Next we try to find a possibility that a consistent non-supersymmetric solution which satisfies $D_{P} \mathcal{W} \neq 0$ with $\partial_{P} V=0$. Actually we find later that there are neither supersymmetric nor non-supersymmetric solutions.

\subsection{Supersymmetric vacua}

In a supersymmetric solution, the equations $D_{\check{a}} \mathcal{W}=0$ and $D_{U} \mathcal{W}=0$ are satisfied. We again impose $\operatorname{Im} U \neq 0$ to find a solution with finite curvature. Actually this configuration is analogous to the case in heterotic string theory compactifications in the presence of $H$-flux ${ }^{2}$.

For simplicity, let us first consider a single modulus model $t^{\check{a}} \equiv t$. In this case the covariant derivative is reduced to

$$
D_{t} \mathcal{W}^{\mathcal{Q}}=\frac{1}{t-\bar{t}}\left(e_{0}(2 t+\bar{t})+3 e_{00}\right)
$$

Then we find

$$
2 t+\bar{t}=-\frac{3 e_{00}}{e_{0}}
$$

where the right-hand side is a real value. This implies the solution $t$ should be real, while this is inadmissible because the curvature (3.9) becomes singular at that point. Thus we find there are no consistent supersymmetric solutions which satisfy $D_{t} \mathcal{W}^{\mathcal{Q}}=0$. In the same way, we also find that there are no consistent solutions of $\mathcal{W}^{\mathcal{Q}}=0$ because $\mathcal{W}^{\mathcal{Q}}=-\left(e_{00}+e_{0} t_{1}\right)-i e_{0} t_{2}$ can be zero if and only if $t_{2}=0$, which gives rise to singular curvature. Then we conclude that there are no supersymmetric solutions in the single modulus model.

Next we study so-called the stu-model given by the three local variables:

$$
\mathcal{F}=\frac{X^{s} X^{t} X^{u}}{X^{0}}, \quad X^{s}=X^{0} s, \quad X^{t}=X^{0} t, \quad X^{u}=X^{0} u
$$

\footnotetext{
${ }^{2}$ Precisely speaking, the condition $\mathrm{d} H \neq 0$ is necessary to see a supersymmetric flux vacua in heterotic theory [48].
} 
We set $X^{0}=1$. The superpotential $\mathcal{W}^{\mathcal{Q}}$, the Kähler potential $K_{+}$, and other functions are described as

$$
\begin{aligned}
\mathcal{W}^{\mathcal{Q}} & =-e_{00}-e_{0 s} s-e_{0 t} t-e_{0 u} u \\
K_{+} & =-\log (-i(s-\bar{s})(t-\bar{t})(u-\bar{u})), \\
\partial_{s} K_{+} & =-\frac{1}{s-\bar{s}}, \quad \partial_{t} K_{+}=-\frac{1}{t-\bar{t}}, \quad \partial_{u} K_{+}=-\frac{1}{u-\bar{u}}, \\
\left(K_{+}\right)^{\check{a} \breve{b}} & =-\operatorname{diag} \cdot\left((s-\bar{s})^{2},(t-\bar{t})^{2},(u-\bar{u})^{2}\right), \\
C_{s t u} & =\frac{i}{(s-\bar{s})(t-\bar{t})(u-\bar{u})}, \\
D_{s} \mathcal{W} & =\frac{U}{s-\bar{s}}\left(e_{00}+e_{0 s} \bar{s}+e_{0 t} t+e_{0 u} u\right), \quad D_{t} \mathcal{W}=\frac{U}{t-\bar{t}}\left(e_{00}+e_{0 s} s+e_{0 t} \bar{t}+e_{0 u} u\right), \\
D_{u} \mathcal{W} & =\frac{U}{u-\bar{u}}\left(e_{00}+e_{0 s} s+e_{0 t} t+e_{0 u} \bar{u}\right), \\
D_{s} D_{t} \mathcal{W} & =\frac{u-\bar{u}}{(s-\bar{s})(t-\bar{t})} U D_{\bar{u}} \overline{\mathcal{W}}^{\mathcal{Q}}, \quad D_{t} D_{u} \mathcal{W}=\frac{s-\bar{s}}{(t-\bar{t})(u-\bar{u})} U D_{\bar{s}} \overline{\mathcal{W}}^{\mathcal{Q}}, \\
D_{u} D_{s} \mathcal{W} & =\frac{t-\bar{t}}{(u-\bar{u})(s-\bar{s})} D_{\bar{t}} \overline{\mathcal{W}}^{\mathcal{Q}} .
\end{aligned}
$$

Expanding $s=s_{1}+i s_{2}, t=t_{1}+i t_{2}$ and $u=u_{1}+i u_{2}$, we rewrite the supersymmetry conditions:

$$
\begin{aligned}
& 0=D_{s} \mathcal{W}^{\mathcal{Q}} \rightarrow\left\{\begin{array}{l}
0=\frac{1}{2 s_{2}}\left(-e_{0 s} s_{2}+e_{0 t} t_{2}+e_{0 u} u_{2}\right) \\
0=-\frac{1}{2 s_{2}}\left(e_{00}+e_{0 s} s_{1}+e_{0 t} t_{1}+e_{0 u} u_{1}\right)
\end{array}\right. \\
& 0=D_{t} \mathcal{W}^{\mathcal{Q}} \rightarrow\left\{\begin{array}{l}
0=\frac{1}{2 t_{2}}\left(e_{0 s} s_{2}-e_{0 t} t_{2}+e_{0 u} u_{2}\right) \\
0=-\frac{1}{2 t_{2}}\left(e_{00}+e_{0 s} s_{1}+e_{0 t} t_{1}+e_{0 u} u_{1}\right)
\end{array}\right. \\
& 0=D_{u} \mathcal{W}^{\mathcal{Q}} \rightarrow\left\{\begin{array}{l}
0=\frac{1}{2 u_{2}}\left(e_{0 s} s_{2}+e_{0 t} t_{2}-e_{0 u} u_{2}\right) \\
0=-\frac{1}{2 u_{2}}\left(e_{00}+e_{0 s} s_{1}+e_{0 t} t_{1}+e_{0 u} u_{1}\right)
\end{array}\right.
\end{aligned}
$$

The solution is given by

$$
-e_{0 s} s_{1}=e_{00}+e_{0 t} t_{1}+e_{0 u} u_{1}, \quad t_{1}, u_{1}: \text { unfixed, } \quad e_{0 s} s_{2}=e_{0 t} t_{2}=e_{0 u} u_{2}=0 .
$$

In order to obtain the finite curvature, we should impose $s_{2} \neq 0, t_{2} \neq 0$ and $u_{2} \neq 0$. This implies $e_{0 s}=e_{0 t}=e_{0 u}=0$ and then $e_{00}=0$. This solution is interpreted as a Calabi-Yau three-fold in the absence of fluxes. In such a configuration the superpotential $\mathcal{W}^{\mathcal{Q}}$ becomes trivial. Thus we conclude that there are no non-trivial solutions to realize supersymmetric flux vacua in the stu-model.

Even though we increase the number of moduli fields $t^{\check{a}}$, we cannot find any consistent solutions to realize supersymmetric flux vacua with the finite curvature as far as we restrict the prepotential in the form as (2.5). Then we have to modify the form (2.5). This will be discussed in the next section. 


\subsection{Non-supersymmetric vacua}

Here we search a non-supersymmetric solution. In this case we have to solve the differential equation $\partial_{P} V_{\mathcal{W}}=0$ itself.

Let us again consider the single modulus model. In this case the functions have already been given in (3.2). The first derivatives of the scalar potential $\partial_{P} V_{\mathcal{W}}(2.2 \mathrm{a})$ are

$$
\begin{aligned}
\mathrm{e}^{-K} \partial_{t} V_{\mathcal{W}} & =\frac{2 e_{0}|U|^{2}}{3}\left(e_{0}(t+2 \bar{t})+3 e_{00}\right)+\frac{2(\operatorname{Im} U)^{2}}{t-\bar{t}}\left(e_{00}+e_{0} \bar{t}\right)\left(e_{0}(2 t+\bar{t})+3 e_{00}\right), \\
\mathrm{e}^{-K} \partial_{U} V_{\mathcal{W}} & =-\operatorname{Re} U\left(1+i \frac{\operatorname{Re} U}{\operatorname{Im} U}\right)\left(-\frac{1}{3}\left|e_{0}(2 t+\bar{t})+3 e_{00}\right|^{2}+\left|e_{00}+e_{0} t\right|^{2}\right) .
\end{aligned}
$$

These two complex equations give four real equations whose solutions are

$$
\operatorname{Re} U=0, \quad \operatorname{Im} U, t_{1}: \text { unfixed, } \quad t_{2}= \pm \frac{3\left(e_{00}+e_{0} t_{1}\right)}{e_{0}} \sqrt{-\frac{1}{5}} .
$$

This is inconsistent with $t_{2} \in \mathbb{R}$. Then we conclude that there are no consistent solutions which satisfy $\partial_{P} V_{\mathcal{W}}=0$ in the search of non-supersymmetric vacua in the single modulus model.

Next we consider the stu-model with functions (5.6). The derivatives of the scalar potential are

$$
\begin{aligned}
& \mathrm{e}^{-K} \partial_{s} V_{\mathcal{W}}=-\frac{2|U|^{2}}{s-\bar{s}}\left(e_{00}+e_{0 s} \bar{s}+e_{0 t} t+e_{0 u} \bar{u}\right)\left(e_{00}+e_{0 s} \bar{s}+e_{0 t} \bar{t}+e_{0 u} u\right) \\
& +\frac{2|U|^{2}}{s-\bar{s}}\left(e_{00}+e_{0 s} \bar{s}+e_{0 t} t+e_{0 u} u\right)\left(e_{00}+e_{0 s} s+e_{0 t} \bar{t}+e_{0 u} \bar{u}\right) \\
& -\frac{(U-\bar{U})^{2}}{2(s-\bar{s})}\left(e_{00}+e_{0 s} \bar{s}+e_{0 t} \bar{t}+e_{0 u} \bar{u}\right)\left(e_{00}+e_{0 s} \bar{s}+e_{0 t} t+e_{0 u} u\right), \\
& \mathrm{e}^{-K} \partial_{t} V_{\mathcal{W}}=-\frac{2|U|^{2}}{t-\bar{t}}\left(e_{00}+e_{0 s} s+e_{0 t} \bar{t}+e_{0 u} \bar{u}\right)\left(e_{00}+e_{0 s} \bar{s}+e_{0 t} \bar{t}+e_{0 u} u\right) \\
& +\frac{2|U|^{2}}{t-\bar{t}}\left(e_{00}+e_{0 s} s+e_{0 t} \bar{t}+e_{0 u} u\right)\left(e_{00}+e_{0 s} \bar{s}+e_{0 t} t+e_{0 u} \bar{u}\right) \\
& -\frac{(U-\bar{U})^{2}}{2(t-\bar{t})}\left(e_{00}+e_{0 s} \bar{s}+e_{0 t} \bar{t}+e_{0 u} \bar{u}\right)\left(e_{00}+e_{0 s} s+e_{0 t} \bar{t}+e_{0 u} u\right), \\
& \mathrm{e}^{-K} \partial_{u} V_{\mathcal{W}}=-\frac{2|U|^{2}}{u-\bar{u}}\left(e_{00}+e_{0 s} \bar{s}+e_{0 t} t+e_{0 u} \bar{u}\right)\left(e_{00}+e_{0 s} s+e_{0 t} \bar{t}+e_{0 u} \bar{u}\right) \\
& +\frac{2|U|^{2}}{u-\bar{u}}\left(e_{00}+e_{0 s} s+e_{0 t} t+e_{0 u} \bar{u}\right)\left(e_{00}+e_{0 s} \bar{s}+e_{0 t} \bar{t}+e_{0 u} u\right) \\
& -\frac{(U-\bar{U})^{2}}{2(u-\bar{u})}\left(e_{00}+e_{0 s} \bar{s}+e_{0 t} \bar{t}+e_{0 u} \bar{u}\right)\left(e_{00}+e_{0 s} s+e_{0 t} t+e_{0 u} \bar{u}\right), \\
& \mathrm{e}^{-K} \partial_{U} V_{\mathcal{W}}=-\frac{\bar{U}(U+\bar{U})}{U-\bar{U}}\left(e_{00}+e_{0 s} \bar{s}+e_{0 t} t+e_{0 u} u\right)\left(e_{00}+e_{0 s} s+e_{0 t} \bar{t}+e_{0 u} \bar{u}\right) \\
& -\frac{\bar{U}(U+\bar{U})}{U-\bar{U}}\left(e_{00}+e_{0 s} s+e_{0 t} \bar{t}+e_{0 u} u\right)\left(e_{00}+e_{0 s} \bar{s}+e_{0 t} t+e_{0 u} \bar{u}\right) \\
& -\frac{\bar{U}(U+\bar{U})}{U-\bar{U}}\left(e_{00}+e_{0 s} s+e_{0 t} t+e_{0 u} \bar{u}\right)\left(e_{00}+e_{0 s} \bar{s}+e_{0 t} \bar{t}+e_{0 u} u\right)
\end{aligned}
$$




$$
+\frac{\bar{U}(U+\bar{U})}{U-\bar{U}}\left(e_{00}+e_{0 s} s+e_{0 t} t+e_{0 u} u\right)\left(e_{00}+e_{0 s} \bar{s}+e_{0 t} \bar{t}+e_{0 u} \bar{u}\right) .
$$

All these equations should vanish to realize a non-supersymmetric solution. Computing them, we obtain two solutions:

$$
\begin{aligned}
& \left\{\begin{array}{c}
\operatorname{Re} U=0, \quad \operatorname{Im} U, s_{1}, t_{1}, u_{1}: \quad \text { unfixed, } \quad-\operatorname{Re}\left(\mathcal{W}^{\mathcal{Q}}\right) \equiv e_{00}+e_{0 s} s_{1}+e_{0 t} t_{1}+e_{0 u} u_{1}, \\
s_{2}=-\frac{\operatorname{Re}\left(\mathcal{W}^{\mathcal{Q}}\right)}{\sqrt{-5} e_{0 s}}, \quad t_{2}=-\frac{\operatorname{Re}\left(\mathcal{W}^{\mathcal{Q}}\right)}{\sqrt{-5} e_{0 t}}, \quad u_{2}=-\frac{\operatorname{Re}\left(\mathcal{W}^{\mathcal{Q}}\right)}{\sqrt{-5} e_{0 u}}
\end{array}\right\} \\
& \left\{\begin{array}{cl}
\operatorname{Im} U, u_{1}, t_{1}, u_{1}, u_{2}: \text { unfixed, }, & \omega \equiv-\frac{1-\sqrt{3} i}{2}, \\
\operatorname{Re} U=i \operatorname{Im} U, \quad s_{1}=-\frac{e_{00}+e_{0 t} t_{1}+e_{0 u} u_{1}}{e_{0 s}}, & s_{2}=-\frac{e_{0 u} u_{2}}{e_{0 s}} \omega^{2}, \quad t_{2}=\frac{e_{0 u} u_{2}}{e_{0 t}} \omega
\end{array}\right\}
\end{aligned}
$$

Both of them are inconsistent. We conclude that there are no consistent solutions to realize nonsupersymmetric flux vacua in the stu-model. In principle, the structures of the equations in multi moduli models are same as the stu-model. Then we also find that there are no non-supersymmetric flux vacua in a generic multi moduli model.

We summarize that there are no consistent solutions to realize four-dimensional spacetime vacua only in the presence of geometric fluxes if the prepotential is restricted to (2.5). It is inevitable to introduce corrections to the prepotential $\mathcal{F}$.

\section{Example 4: another model on $S U(3)$-structure manifold}

Since we could not find any consistent solutions in section 5, we have to introduce a deformation in the prepotential $\mathcal{F}$ in the following way ${ }^{3}$ :

$$
\mathcal{F}(X)=D_{\check{a} \check{b} \check{c}} \frac{X^{\check{a}} X^{\check{b}} X^{\check{c}}}{X^{0}}+\widetilde{\mathcal{F}}(X),
$$

where $\widetilde{\mathcal{F}}(X)$ is also a holomorphic function of the projective coordinates of degree two. Here we focus on a single modulus model $t^{\check{a}} \equiv t$. The prepotential $\mathcal{F}$ and the Kähler potential are given by

$$
\begin{aligned}
\mathcal{F} & =\frac{X^{t} X^{t} X^{t}}{X^{0}}+\widetilde{\mathcal{F}}(X), \quad X^{t}=X^{0} t, \\
K_{+} & =-\log \left(-i\left(t-\bar{t}^{3}\right)+i N\right), \\
N & \equiv \widetilde{\mathcal{F}}_{0}-\overline{\widetilde{\mathcal{F}}}_{0}+\bar{t} \widetilde{\mathcal{F}}_{t}-t \overline{\widetilde{\mathcal{F}}}_{t}, \quad \partial_{t} N \equiv \widetilde{\mathcal{F}}_{0 t}+\bar{t} \widetilde{\mathcal{F}}_{t t}-\overline{\widetilde{\mathcal{F}}}_{t} .
\end{aligned}
$$

For a minimal setup we introduce the deformed term $\widetilde{\mathcal{F}}$ in the following form:

$$
\widetilde{\mathcal{F}}=N_{1} \frac{\left(X^{t}\right)^{4}}{\left(X^{0}\right)^{2}} .
$$

\footnotetext{
${ }^{3}$ Insertion of the corrections is also discussed in [49].
} 
Then the function $N$ and its derivatives are

$$
\begin{aligned}
N & =-2\left(N_{1} t^{4}-\bar{N}_{1} \bar{t}^{4}-2 N_{1} t^{3} \bar{t}+2 \bar{N}_{1} t \bar{t}^{3}\right), \\
\partial_{t} N & =-4\left(2 N_{1} t^{3}-3 N_{1} t^{2} \bar{t}+\bar{N}_{1} \bar{t}^{3}\right) .
\end{aligned}
$$

The function $N$ gives a consistent solution of $D_{t} \mathcal{W}^{\mathcal{Q}}=0$ :

$$
\begin{aligned}
t_{1 *} & =-\frac{2 e_{00}}{e_{0}}, \quad t_{2 *}=0, \quad \operatorname{Re} U_{*}=0, \\
\mathcal{W}_{*}^{\mathcal{Q}} & =e_{00}, \\
M_{*} & =-\frac{64 i\left(e_{00}\right)^{4} \operatorname{Im} N_{1}}{\left(e_{0}\right)^{4}}, \quad\left(K_{+}\right)_{*}=-\log \left(-\frac{64\left(e_{00}\right)^{4} \operatorname{Im} N_{1}}{\left(e_{0}\right)^{4}}\right), \\
\left.R_{t t \bar{t}}^{t}\right|_{*} & =\frac{\left(e_{0}\right)^{2}}{256} \frac{832\left(e_{00}\right)^{2}\left(\operatorname{Im} N_{1}\right)^{2}-144 e_{00} e_{0} \operatorname{Re} N_{1}+576\left(e_{00}\right)^{2}\left(\operatorname{Re} N_{1}\right)^{2}+9\left(e_{0}\right)^{2}}{\left(e_{00}\right)^{4} \operatorname{Im} N_{1}} .
\end{aligned}
$$

This is indeed a solution which gives the finite curvature. We have to set $\operatorname{Im} N_{1}$ to be negative definite, otherwise the Kähler potential $K_{+}$is ill-defined. The scalar potential is evaluated:

$$
V_{*}=-3 \mathrm{e}^{K}\left|\mathcal{W}_{*}\right|^{2}=\frac{1}{\left[\operatorname{Re}\left(\mathcal{C} \mathcal{G}_{0}\right)\right]^{2}} \frac{3\left(e_{0}\right)^{4}}{16\left(e_{00}\right)^{2} \operatorname{Im} N_{1}} .
$$

Due to the condition $\operatorname{Im} N_{1}<0, V_{*}$ provides the negative cosmological constant. In order to satisfy the supergravity approximation, the value $\operatorname{Re}\left(\mathcal{C G}_{0}\right)$ should be very large. This is nothing but the solution to realize a supersymmetric AdS vacuum in the compactification on the $S U(3)$-structure manifold.

We also find that this scalar potential and the curvature tensor go to infinity when we take the limit $N_{1} \rightarrow 0$. The geometric flux charges deform the internal space. This is the reason why we could not find any solutions in section 5. This result again differs from that of [44]. If we set the torsion charge $e_{0}$ to be zero, the internal manifold is reduced to a Calabi-Yau three-fold with $H$-flux charges $e_{00}$. Here we cannot take the large volume limit (2.5) caused by the existence of $H$-flux. Then the deformation (6.1) is inevitable. In this case the cosmological constant vanishes and a Minkowski vacuum appears. This is consistent with that of [44].

\section{Summary and discussions}

In this paper we studied supersymmetric vacua in four-dimensional $\mathcal{N}=1$ supergravity derived from type IIA string theory compactified on generalized geometries with $S U(3) \times S U(3)$ structures. We started with a generic form of the scalar potential in $\mathcal{N}=1$ supergravity which contains a superpotential and D-terms. The superpotential is built from two parts; one is given by RamondRamond flux charges, the other by (non)geometric flux charges. We referred to the former as the Ramond-Ramond flux superpotential, and to the latter as the (non)geometric flux superpotential. 
To make the discussion clear, we first addressed a simple model with a prepotential given by the intersection number in a way analogous to a model derived from a compactification on CalabiYau three-fold in the large volume limit. We obtained two supersymmetric vacua characterized by discriminants of the superpotentials. If the discriminants of the Ramond-Ramond flux superpotential and of the (non)geometric flux superpotential are positive, a supersymmetric AdS vacuum is realized. The cosmological constant is given by the square root of the discriminant of the superpotential. This situation is akin to flux vacua attractors in type IIB theory. On the other hand, if both of these two discriminants are negative, the cosmological constant vanishes and a supersymmetric Minkowski vacuum appears.

Next we explored consistent supersymmetric vacua in the absence of Ramond-Ramond flux charges. In a simple model on generalized geometry with $S U(3) \times S U(3)$ structures, we again obtained a supersymmetric AdS vacuum with a negative cosmological constant. If the nongeometric flux charges are turned off in a situation where the prepotential is described only in terms of the intersection number, there exist neither supersymmetric nor non-supersymmetric solutions. Then we analyzed another model which has a prepotential with a deformation term, obtaining a consistent supersymmetric AdS vacuum. This implies that a model compactified on an $S U(3)$-structure manifold with torsion in the absence of Ramond-Ramond flux charges differs from a model given by Calabi-Yau compactification in the large volume limit.

There are four interesting issues which deserve further study in flux compactification scenarios on generalized geometries: (i) In this paper there is no way to fix the real part of the modulus $U$ in the supersymmetric AdS vacua, partly because we restricted the number of complex variables $U^{\check{I}}$ to one. If one incorporates more than one variable, there might appear a richer structure in various functions, especially in the second derivatives of the Kähler potential. In addition, it is also worth considering non-perturbative corrections to stabilize all moduli. (ii) In a generic configuration with Ramond-Ramond flux charges and nongeometric flux charges, we restricted the form of the prepotential governing the chiral scalar variables $t^{\check{a}}$ in the same way as one does for the Calabi-Yau compactification in the large volume limit. This corresponds to a model compactified on a parallelizable twisted torus. One should also consider models arising from more generic prepotentials to understand lowerdimensional effective theories of compactifications on (non)geometric string backgrounds. (iii) The Bianchi identity of form fluxes should also be considered seriously to study consistent configurations of D-branes and orientifold planes wrapped on the internal space [12]. (iv) Duality transformations on generalized geometries are crucial in elucidating the stringy origin of nongeometric fluxes in a more explicit way [16]. This way also be helped by use of doubled space formalism [20, 22-24,50]. Duality transformations and nongeometric compactifications may also ultimately lead to a complete classification of lower-dimensional gauged supergravities which are not derived from higher-dimensional supergravities compactified on conventional geometries. 


\section{Acknowledgements}

The author would like to thank Tohru Eguchi for illuminating discussions in the early stages of this work.

\section{Appendix}

\section{A Supersymmetry parameters}

We consider type IIA string theory compactified on generalized geometries. Let us assume that tendimensional metric is given by $\mathrm{d} s_{10}^{2}=\mathrm{e}^{2 A} g_{\mu \nu} \mathrm{d} x^{\mu} \mathrm{d} x^{\nu}+g_{m n} \mathrm{~d} y^{m} \mathrm{~d} y^{n}$, where $g_{\mu \nu}$ and $g_{m n}$ are the metric of the four-dimensional spacetime $\mathcal{M}_{3,1}$ and that of the six-dimensional space $\mathcal{N}$, respectively. We also introduced a warp factor $A$. For simplicity, the warp factor is a constant. The ten-dimensional supersymmetry parameters $\epsilon^{1}$ and $\epsilon^{2}$ are split into two parts:

$$
\epsilon^{1}=\varepsilon_{1} \otimes \bar{a} \eta_{-}^{1}+\varepsilon^{1} \otimes a \eta_{+}^{1} \quad \epsilon^{2}=\varepsilon_{2} \otimes b \eta_{+}^{2}+\varepsilon^{2} \otimes \bar{b} \eta_{-}^{2} .
$$

Here $\varepsilon_{\mathcal{A}}$ with indices $\mathcal{A}=1,2$ are Weyl fermions as the four-dimensional supersymmetry parameters whose charge conjugates are $\varepsilon_{\mathcal{A}}^{c} \equiv \varepsilon^{\mathcal{A}}$. The $\eta_{ \pm}^{\mathcal{A}}$ are $S U(4)$ Weyl spinors in the six-dimensional internal space with $\left(\eta_{ \pm}^{\mathcal{A}}\right)^{c}=\left(\eta_{ \pm}^{\mathcal{A}}\right)^{*}$. The chirality of $\epsilon^{1}\left(\epsilon^{2}\right)$ in type IIA theory is negative (positive) $[7,15]$. The two complex scale parameters $a$ and $b$ are normalization factors $[12,15]$ with $|a|^{2}+|b|^{2}=c_{+}$and $|a|^{2}-|b|^{2}=c_{-}$. Without loss of generality we can set $c_{+}=1$. Indeed, the coefficients $a$ and $b$ would be related to the warp factor $A$ in $\mathcal{N}=1$ vacua [6]. In order to obtain $\mathcal{N}=2$ and $\mathcal{N}=1$ supersymmetries in four-dimensional spacetime, the $S U(4)$ spinors are reduced to $S U(3)$ invariant spinors which are interpreted as Killing spinors on $\mathcal{M}$.

\section{B Generalized geometries with $S U(3) \times S U(3)$ structures}

\section{B.1 Generalized complex structures and pure spinors}

In the splitting of type IIA supersymmetry parameters (A.1), there emerges a pair of $S U(3)$ invariant spinors $\eta_{+}^{1}$ and $\eta_{+}^{2}$. These two spinors are related to each other via the expression [7]

$$
\eta_{+}^{2}=c_{\|} \eta_{+}^{1}+c_{\perp}\left(v+i v^{\prime}\right)^{m} \gamma_{m} \eta_{-}^{1}, \quad\left|c_{\|}\right|^{2}+\left|c_{\perp}\right|^{2}=1
$$

where $\gamma_{m}$ is the Cliff(6) gamma matrix acting on $\eta_{ \pm}^{\mathcal{A}}$. The two vectors $v$ and $v^{\prime}$ are defined by the bilinear form of the spinors as $\left(v-i v^{\prime}\right)^{m}=\eta_{+}^{1 \dagger} \gamma^{m} \eta_{-}^{2}$. The coefficients $c_{\|}$and $c_{\perp}$ depend on the coordinates of the internal space $\mathcal{M}$. This pair of spinors defines a pair of $S U(3)$-structure groups, 
where the structure group is the group in which the transition functions of the tangent bundle $T \mathcal{M}$ take their values. If $c_{\perp}=0$ at any point on $\mathcal{M}$, the two spinors coincide with each other and the structure group is reduced to a single $S U(3)$. As usual one defines the almost complex structures in terms of the $S U(3)$ invariant spinors as $\left(J^{\mathcal{A}}\right)^{m}{ }_{n}=-2 i \eta_{+}^{\mathcal{A} \dagger} \gamma^{m}{ }_{n} \eta_{+}^{\mathcal{A}}$. If $\eta_{+}^{1}=\eta_{+}^{2}$ at any points, the almost complex structures $J^{1}$ also coincides with $J^{2}$. We refer to $\mathcal{M}$ with a single almost complex structure as a manifold with a single $S U(3)$-structure, or simply an $S U(3)$-structure manifold. On the other hand, if $c_{\perp} \neq 0$ at some points on $\mathcal{M}$, there exists a pair of almost complex structures on $\mathcal{M}$, and we refer to this as a manifold with a pair of $S U(3)$-structures.

To go beyond an ordinary almost complex structure, one considers a space $T \mathcal{M} \oplus T^{*} \mathcal{M}$ and introduces generalized almost complex structures $\mathcal{J}_{ \pm}$which give rise to a mapping $\mathcal{J}_{ \pm}: T \mathcal{M} \oplus T^{*} \mathcal{M} \rightarrow$ $T \mathcal{M} \oplus T^{*} \mathcal{M}$. Since the basis of the space $T \mathcal{M} \oplus T^{*} \mathcal{M}$ is given by $\left\{\mathrm{d} x^{m} \wedge, \iota_{\partial_{n}}\right\}$, the signature of this space is $(6,6)$. Let us first describe $\mathcal{J}_{ \pm}$by means of sections of spinor bundles associated with $T \mathcal{M} \oplus T^{*} \mathcal{N}$ :

$$
\mathcal{J}_{ \pm \Sigma}^{\Lambda}=\left\langle\operatorname{Re} \Phi_{ \pm}, \Gamma_{\Sigma}^{\Lambda} \operatorname{Re} \Phi_{ \pm}\right\rangle .
$$

Here we introduced complex $S U(3,3)$ invariant spinors $\Phi_{ \pm}$, the $\operatorname{Cliff}(6,6)$ gamma matrix $\Gamma^{\Lambda}$ and its antisymmetrized product $\Gamma^{\Lambda \Sigma}=\frac{1}{2}\left(\Gamma^{\Lambda} \Gamma^{\Sigma}-\Gamma^{\Sigma} \Gamma^{\Lambda}\right)$, where the indices are raised and lowered with the $S O(6,6)$ invariant metric $L^{\Lambda \Sigma}$. Since the irreducible representation of $\operatorname{Spin}(6,6)$ spinor is MajoranaWeyl, $\Phi_{+}\left(\Phi_{-}\right)$can be assigned to a Weyl spinor with positive (negative) chirality. The two Weyl spinor bundles on $T \mathcal{M} \oplus T^{*} \mathcal{M}$ are isomorphic to the spaces of even/odd forms $\wedge^{\text {even/odd }} T^{*} \mathcal{M}$. The $S U(3,3)$ invariant Weyl spinors $\Phi_{ \pm}$are pure since they are annihilated by half of the Cliff $(6,6)$ gamma matrices $\Gamma_{\Lambda}$. Due to the isomorphism, the bracket in (B.2) can be expressed by the Mukai pairing

$$
\left\langle A_{p}, B_{q}\right\rangle \equiv\left[A_{p} \wedge \lambda\left(B_{q}\right)\right]_{\text {top form }}, \quad \lambda\left(B_{q}\right) \equiv(-1)^{\left[\frac{q}{2}\right]} B_{q},
$$

where $A_{p}$ and $B_{q}$ are arbitrary $p$-form and $q$-form, respectively. When a generalized almost complex structure $\mathcal{J}$ is defined, we refer to the space $\mathcal{M}$ as a generalized (almost complex) geometry. The two Weyl spinors $\Phi_{ \pm}$can be described in terms of the supersymmetry parameters $\eta_{ \pm}^{\mathcal{A}}$ in (A.1) [7]:

$$
\Phi_{ \pm}=\mathrm{e}^{-B} \Phi_{ \pm}^{0}, \quad \Phi_{ \pm}^{0}=8 \eta_{+}^{1} \otimes \eta_{ \pm}^{2 \dagger} \equiv \sum_{k=0}^{6} \frac{1}{k !}\left(\eta_{ \pm}^{2 \dagger} \gamma_{m_{1} \cdots m_{k}} \eta_{+}^{1}\right) \gamma^{m_{k} \cdots m_{1}}
$$

where $B$ is a two-form. Actually the bilinear forms $\Phi_{ \pm}^{0}$ satisfy the following differential equations in $\mathcal{N}=1$ vacua derived from type IIA theory (see $[6,12,15])$

$$
\begin{aligned}
& \mathrm{e}^{-2 A+\phi}(\mathrm{d}-H \wedge)\left(\mathrm{e}^{2 A-\phi} \Phi_{+}^{0}\right)=-2 \bar{\mu} \operatorname{Re} \Phi_{-}^{0}, \\
& \mathrm{e}^{-2 A+\phi}(\mathrm{d}-H \wedge)\left(\mathrm{e}^{2 A-\phi} \overline{\Phi_{-}^{0}}\right)=-3 i \operatorname{Im}\left(\bar{\mu} \overline{\Phi_{+}^{0}}\right)+\frac{1}{16} \mathrm{e}^{\phi}\left[c_{-} F^{\text {even }}+i c_{+} * \lambda\left(F^{\text {even }}\right)\right],
\end{aligned}
$$

where $F^{\text {even }}=F_{0}+F_{2}+F_{4}+F_{6}$ is a sum of the Ramond-Ramond forms. The $\mu$ gives the cosmological constant $\Lambda=-|\mu|^{2}$ in four-dimensional spacetime. Note that the structure group of the generalized geometry is $S U(3) \times S U(3)$ if $c_{\perp} \neq 0$ at some points on $\mathcal{M}$, or $S U(3)$ if $c_{\perp}=0$ at any points on $\mathcal{M}$. 
It is also known that the spaces of $\Phi_{ \pm}$are given by special Kähler geometries of local type [7]. This implies that the generalized geometry has the moduli space given by the product of the two Hodge-Kähler geometries whose Kähler potentials $K_{ \pm}$are $^{4}$

$$
K_{+}=-\log i \int_{\mathcal{M}}\left\langle\Phi_{+}, \bar{\Phi}_{+}\right\rangle, \quad K_{-}=-\log i \int_{\mathcal{M}}\left\langle\Phi_{-}, \bar{\Phi}_{-}\right\rangle .
$$

We assign the special Kähler geometries given by $\Phi_{ \pm}$to $\mathcal{M}_{ \pm}$, respectively. One can introduce projective coordinates $X^{A}$ and a prepotential $\mathcal{F}$ on $\mathcal{M}_{+}$(and projective coordinates $Z^{I}$ and a prepotential $\mathcal{G}$ on $\mathcal{M}_{-}$). The prepotentials $\mathcal{F}$ and $\mathcal{G}$ are functions of holomorphic and homogeneous of degree two in the projective coordinates. Since the two Weyl spinors $\Phi_{ \pm}$are isomorphic to the even and odd forms, they are expanded in terms of basis forms:

$$
\Phi_{+}=X^{A} \omega_{A}-\mathcal{F}_{A} \widetilde{\omega}^{A}, \quad \Phi_{-}=Z^{I} \alpha_{I}-\mathcal{G}_{I} \beta^{I},
$$

where $\omega_{A}$ and $\widetilde{\omega}^{A}$ are even real basis forms (i.e., zero-, two-, four- and six-forms), while $\alpha_{I}$ and $\beta^{I}$ are odd real basis forms (one-, three- and five-forms). The coefficients are interpreted as the projective coordinates and derivatives of the prepotentials.

\section{B.2 Property of Special Kähler geometries}

The projective coordinates and the prepotentials on the special Kähler geometries are described in terms of period integrals of the Mukai pairing:

$$
\begin{aligned}
X^{A} & =\int_{\mathcal{M}}\left\langle\Phi_{+}, \widetilde{\omega}^{A}\right\rangle, & \mathcal{F}_{A} & =\frac{\partial \mathcal{F}}{\partial X^{A}}=\int_{\mathcal{M}}\left\langle\Phi_{+}, \omega_{A}\right\rangle, \\
Z^{I} & =\int_{\mathcal{M}}\left\langle\Phi_{-}, \beta^{I}\right\rangle, & \mathcal{G}_{I} & =\frac{\partial \mathcal{G}}{\partial Z^{I}}=\int_{\mathcal{M}}\left\langle\Phi_{-}, \alpha_{I}\right\rangle,
\end{aligned}
$$

where we used the symplectic structure among the basis forms

$$
\begin{aligned}
& \left(\begin{array}{ll}
\int_{\mathcal{M}}\left\langle\omega_{A}, \omega_{B}\right\rangle & \int_{\mathcal{M}}\left\langle\omega_{A}, \widetilde{\omega}^{B}\right\rangle \\
\int_{\mathcal{M}}\left\langle\widetilde{\omega}^{A}, \omega_{B}\right\rangle & \int_{\mathcal{M}}\left\langle\widetilde{\omega}^{A}, \widetilde{\omega}^{B}\right\rangle
\end{array}\right)=\left(\begin{array}{cc}
0 & \delta_{A}^{B} \\
-\delta^{A}{ }_{B} & 0
\end{array}\right), \quad A, B=0,1, \ldots, b^{+}, \\
& \left(\begin{array}{ll}
\int_{\mathcal{M}}\left\langle\alpha_{I}, \alpha_{J}\right\rangle & \int_{\mathcal{M}}\left\langle\alpha_{I}, \beta^{J}\right\rangle \\
\int_{\mathcal{M}}\left\langle\beta^{I}, \alpha_{J}\right\rangle & \int_{\mathcal{M}}\left\langle\beta^{I}, \beta^{J}\right\rangle
\end{array}\right)=\left(\begin{array}{cc}
0 & \delta_{I}^{J} \\
-\delta^{I}{ }_{J} & 0
\end{array}\right), \quad I, J=0,1, \ldots, b^{-} .
\end{aligned}
$$

Then the Kähler potentials $K_{ \pm}$in (B.6) are described as

$$
K_{+}=-\log i\left(\bar{X}^{A} \mathcal{F}_{A}-X^{A} \overline{\mathcal{F}}_{A}\right), \quad K_{-}=-\log i\left(\bar{Z}^{I} \mathcal{G}_{I}-Z^{I} \overline{\mathcal{G}}_{I}\right)
$$

\footnotetext{
${ }^{4}$ Here these Kähler potentials are reduced to functions in four-dimensional spacetime [7].
} 
We can choose a set of local coordinate frames of $\mathcal{M}_{ \pm}$as $X^{A}=\left(X^{0}, X^{a}\right)=\left(X^{0}, X^{0} t^{a}\right)$ and $Z^{I}=$ $\left(Z^{0}, Z^{i}\right)=\left(Z^{0}, Z^{0} z^{i}\right)$, where $A, B, C, \ldots$ and $a, b, c, \ldots$ are projective and local coordinate indices, respectively. The properties of their functions include

$$
\begin{aligned}
& \partial_{a} \equiv \frac{\partial}{\partial t^{a}}, \quad D_{c} \equiv \partial_{c}+\partial_{c} K_{+}, \\
& \mathcal{F}_{A}=\mathcal{N}_{A B} X^{B}, \quad D_{a} \mathcal{F}_{B}=\overline{\mathcal{N}}_{B C} D_{a} X^{C}, \quad\left(K_{+}\right)_{a \bar{b}}=\partial_{a} \partial_{\bar{b}} K_{+}, \\
& \mathrm{e}^{K_{+}}\left(K_{+}\right)^{a \bar{b}} D_{a} X^{C} D_{\bar{b}} \bar{X}^{D}=-\frac{1}{2}\left[(\operatorname{Im} \mathcal{N})^{-1}\right]^{C D}-\mathrm{e}^{K_{+}} \bar{X}^{C} X^{D}, \\
& C_{a b c}=\mathrm{e}^{K_{+}}\left(\partial_{a} X^{A}\right)\left(\partial_{b} X^{B}\right)\left(\partial_{c} X^{C}\right) \mathcal{F}_{A B C}(X), \quad \mathcal{F}_{A B C}=\frac{\partial^{3} \mathcal{F}}{\partial X^{A} \partial X^{B} \partial X^{C}},
\end{aligned}
$$

where $\mathcal{N}_{A B}$ is the period matrix on the moduli space $\mathcal{M}_{+}$. Here $D_{c}$ is the Kähler covariant derivative. Details of the special Kähler geometry can be found, for instance, in $[15,17,45]$. Notice that $C_{a b c}$ is a totally symmetric Kähler covariantly holomorphic tensor on $\mathcal{M}_{+}$.

\section{B.3 (Non)geometric flux charges}

Once the NS-NS three-form flux $H$ is incorporated into the six-dimensional internal space $\mathcal{M}$, this space is no longer a Calabi-Yau three-fold ${ }^{5}$. Although this flux does not modify the $S U(3)$-structure group, a non-constant dilaton, a warp factor and torsion are induced. We call them geometric fluxes.

In the case of the generalized geometry with $S U(3) \times S U(3)$ structures, we should introduce a set of charges $p_{I}{ }^{A}$ and $q^{I A}[7]$, called the charges of "nongeometric fluxes" as well as geometric electricand magnetic-charges $e_{I A}$ and $m_{A}{ }^{I}[22]$. One has to generalize the exterior derivative $\mathrm{d}$ to $\mathcal{D}$ in the following way:

$$
\begin{array}{rlrl}
\mathcal{D} \omega_{A} & \sim m_{A}{ }^{I} \alpha_{I}-e_{I A} \beta^{I}, & \mathcal{D} \widetilde{\omega}^{A} \sim-q^{I A} \alpha_{I}+p_{I}{ }^{A} \beta^{I}, \\
\mathcal{D} \alpha_{I} \sim p_{I}{ }^{A} \omega_{A}+e_{I A} \widetilde{\omega}^{A}, & \mathcal{D} \beta^{I} \sim q^{I A} \omega_{A}+m_{A}{ }^{I} \widetilde{\omega}^{A},
\end{array}
$$

where $\sim$ means equality up to terms vanishing inside the Mukai pairing (B.3) in computations of the Kähler potentials and superpotentials. Here $\mathcal{D}$ is described as $\mathcal{D} \equiv \mathrm{d}-H^{\mathrm{fl}} \wedge-f \cdot-Q \cdot-R$, where $H^{\mathrm{fl}}$ is the NS-NS three-form flux ${ }^{6} H^{\mathrm{fl}} \equiv H-\mathrm{d} B$, while $f, Q$ and $R$ are called the (non)geometric fluxes acting on an arbitrary $k$-form $C$ as $(f \cdot C)_{m_{1} \cdots m_{k+1}} \equiv f_{\left[m_{1} m_{2}\right.}^{a} C_{\left.|a| m_{3} \cdots m_{k+1}\right]},(Q \cdot C)_{m_{1} \cdots m_{k-1}} \equiv$ $Q^{a b}{ }_{\left[m_{1}\right.} C_{\left.|a b| m_{2} \cdots m_{k-1}\right]}$ and $\left(R\llcorner C)_{m_{1} \cdots m_{k-3}} \equiv R^{a b c} C_{a b c m_{1} \cdots m_{k-3}}\right.$. Actually the geometric flux $f$ gives a non-trivial structure constant in gauged supergravity via the Scherk-Schwarz compactifications [18], while the fluxes $Q$ and $R$ provide the nongeometric string backgrounds [22] via duality transformations in string theory.

\footnotetext{
${ }^{5}$ In a very restricted case, the internal space becomes a warped Calabi-Yau manifold. Such a geometry appears in type IIB theory flux compactification scenario [10,38].

${ }^{6}$ The cohomology of the $S U(3)$-structure manifold defines the topological indices such as the Dirac index, the Euler characteristics and the Hirzebruch signature [51].
} 
Imposing the nilpotency $\mathcal{D}^{2}=0$, we obtain a set of relations among the (non)geometric flux charges:

$$
\begin{aligned}
& 0=q^{I A} m_{A}{ }^{J}-m_{A}{ }^{I} q^{I A}, \quad 0=p_{I}{ }^{A} e_{A J}-e_{I A} p_{J}{ }^{A}, \quad 0=p_{I}{ }^{A} m_{A}{ }^{J}-e_{I A} q^{J A}, \\
& 0=q^{I A} p_{I}^{B}-p_{I}^{A} q^{I B}, \quad 0=m_{A}^{I} e_{I B}-e_{I A} m_{B}{ }^{I}, \quad 0=m_{A}{ }^{I} p_{I}{ }^{B}-e_{I A} q^{I B} .
\end{aligned}
$$

\section{Type IIA theory compactified on generalized geometry}

We analyze four-dimensional supergravity compactified on the generalized geometry with $S U(3) \times$ $S U(3)$ structures by using the notation and conventions in [15]. First we construct $\mathcal{N}=1$ Kähler potential, superpotential and D-terms in the language of $\mathcal{N}=2$ theory. Then we truncate physical degrees of freedom via $\mathrm{O} 6$ orientifold projection.

$\mathcal{N}=2$ Killing prepotentials are useful to derive the superpotential and the D-terms. Here we briefly review the works $[7,15]$. The Killing prepotentials $\mathcal{P}^{x}$ appear in supersymmetry variations of four-dimensional gravitinos $\psi_{\mathcal{A} \mu}$ as

$$
\begin{aligned}
\delta \psi_{\mathcal{A} \mu} & =\nabla_{\mu} \varepsilon_{\mathcal{A}}-S_{\mathcal{A B}} \gamma_{\mu}^{(4)} \varepsilon^{\mathcal{B}}+\ldots \\
S_{\mathcal{A B}} & =\frac{i}{2} \mathrm{e}^{\frac{K_{+}}{2}}\left(\sigma_{x}\right)_{\mathcal{A}} \epsilon_{\mathcal{B C}} \mathcal{P}^{x}=\frac{i}{2} \mathrm{e}^{\frac{K_{+}}{2}}\left(\begin{array}{cc}
\mathcal{P}^{1}-i \mathcal{P}^{2} & -\mathcal{P}^{3} \\
-\mathcal{P}^{3} & -\mathcal{P}^{1}-i \mathcal{P}^{2}
\end{array}\right)
\end{aligned}
$$

where dots indicate irrelevant parts which do not contribute to the superpotential. Here $\gamma_{\mu}^{(4)}$ is the Dirac gamma matrix in four dimensions, $\left(\sigma_{x}\right)_{\mathcal{A}}{ }^{\mathcal{B}}$ with $x=1,2,3$ are the $S U(2)$ Pauli matrices, and $\epsilon_{\mathcal{A B}}$ is the $S U(2)$ invariant metric utilized to raise and lower indices $\mathcal{A}$. Explicit forms of the Killing prepotentials $\mathcal{P}^{x}$ are written in terms of the Weyl spinors $\Phi_{ \pm}$and the Ramond-Ramond field strength $G$. In the case of compactifications on the generalized geometry with $S U(3) \times S U(3)$ structures, these are given as follows ${ }^{7}$ :

$$
\begin{aligned}
\mathcal{P}^{1}-i \mathcal{P}^{2} & =2 \mathrm{e}^{\frac{K_{-}}{2}+\varphi} \int_{\mathcal{M}}\left\langle\Phi_{+}, \mathcal{D} \Phi_{-}\right\rangle \\
& =2 \mathrm{e}^{\frac{K_{-}}{2}+\varphi}\left[\left(Z^{I} e_{I A}-\mathcal{G}_{I} m_{A}{ }^{I}\right) X^{A}+\left(Z^{I} p_{I}{ }^{A}-\mathcal{G}_{I} q^{I A}\right) \mathcal{F}_{A}\right] \\
\mathcal{P}^{1}+i \mathcal{P}^{2} & =2 \mathrm{e}^{\frac{K_{-}}{2}+\varphi} \int_{\mathcal{M}}\left\langle\Phi_{+}, \mathcal{D} \bar{\Phi}_{-}\right\rangle \\
& =2 \mathrm{e}^{\frac{K_{-}}{2}+\varphi}\left[\left(\bar{Z}^{I} e_{I A}-\overline{\mathcal{G}}_{I} m_{A}^{I}\right) X^{A}+\left(\bar{Z}^{I} p_{I}{ }^{A}-\overline{\mathcal{G}}_{I} q^{I A}\right) \mathcal{F}_{A}\right] \\
\mathcal{P}^{3} & =-\frac{1}{\sqrt{2}} \mathrm{e}^{2 \varphi} \int_{\mathcal{M}}\left\langle\Phi_{+}, G\right\rangle \\
& =\mathrm{e}^{2 \varphi}\left[\left(e_{\mathrm{RR} A}-\xi^{I} e_{I A}+\widetilde{\xi}_{I} m_{A}^{I}\right) X^{A}-\left(m_{\mathrm{RR}}^{A}+\xi^{I} p_{I}{ }^{A}-\widetilde{\xi}_{I} q^{I A}\right) \mathcal{F}_{A}\right] .
\end{aligned}
$$

\footnotetext{
${ }^{7}$ For detailed discussions, see [7] for the case of generalized geometry with a single $S U(3)$-structure, or [7] for that of generalized geometry with $S U(3) \times S U(3)$ structures
} 
If the six-dimensional internal space is a generalized geometry with a single $S U(3)$-structure, the generalized differential operator $\mathcal{D}$ in $(\mathrm{C} .2)$ is reduced to $\mathrm{d}_{H^{\mathrm{f}}}$.

If localized D-branes are absent, it is convenient to define the Ramond-Ramond field strength $G$ as a modification of the field strength $F^{\text {even }}$ multiplied with the exponent of the B-field $[7,53]$ :

$$
F_{n}^{\text {even }}=\left(\mathrm{e}^{B} G\right)_{n}=\mathrm{d} C_{n-1}-H \wedge C_{n-3}, \quad C=\mathrm{e}^{B} A, \quad(\mathrm{~d}-H \wedge) F^{\text {even }}=0 .
$$

In the generalized geometry with $S U(3) \times S U(3)$ structures in the democratic description [53], the Ramond-Ramond field strength $G$ is given in terms of the generalized differential operator $\mathcal{D}$ as

$$
G \equiv G_{0}+G_{2}+G_{4}+G_{6}=G^{\mathrm{fl}}+\mathcal{D} A,
$$

where $G^{\mathrm{fl}}$ and $A$ are the intrinsic part of the field strength and the potential, respectively. Both of them are expanded in terms of the basis of forms as ${ }^{8}$

$$
G^{\mathrm{fl}}=\sqrt{2}\left(m_{\mathrm{RR}}^{A} \omega_{A}-e_{\mathrm{RR} A} \widetilde{\omega}^{A}\right), \quad A=\sqrt{2}\left(\xi^{I} \alpha_{I}-\widetilde{\xi}_{I} \beta^{I}\right)
$$

where $e_{\mathrm{RR} A}$ and $m_{\mathrm{RR}}^{A}$ are electric- and magnetic-charges of the Ramond-Ramond fluxes, respectively. The fields $\xi^{I}$ and $\widetilde{\xi}_{I}$ appear as scalar fields in four dimensions.

Let us elaborate the superpotential. The $\mathcal{N}=1$ supersymmetry parameter $\varepsilon$ is defined by the linear combination of the two $\mathcal{N}=2$ supersymmetry parameters in the following way:

$$
\varepsilon=\bar{n}^{\mathcal{A}} \varepsilon_{\mathcal{A}}
$$

where $\bar{n}^{\mathcal{A}}=(a, \bar{b})$ is a two component vector given by the coefficients $a$ and $b$ in (A.1). In the same way as the linear combination (C.6), the $\mathcal{N}=2$ gravitinos are also linearly combined into the $\mathcal{N}=1$ gravitino as $\psi_{\mu}=\bar{n}^{\mathcal{A}} \psi_{\mathcal{A} \mu}$. Then the $\mathcal{N}=1$ supersymmetry variation is described in terms of the linear combination of the $\mathcal{N}=2$ variations (C.1) in such a way as $\delta \psi_{\mu}=\nabla_{\mu} \varepsilon-\bar{n}^{\mathcal{A}} S_{\mathcal{A B}} n^{* \mathcal{B}} \gamma_{\mu}^{(4)} \varepsilon^{c}$. Since this form is generically expressed as $\delta \psi_{\mu}=\nabla_{\mu} \varepsilon-\mathrm{e}^{\frac{K}{2}} \mathcal{W} \gamma_{\mu}^{(4)} \varepsilon^{c}$ [15], we obtain an explicit form of the superpotential $\mathcal{W}$ as

$$
\mathrm{e}^{\frac{K}{2}} \mathcal{W}=\bar{n}^{\mathcal{A}} S_{\mathcal{A B}} n^{* \mathcal{B}}=\frac{i}{2} \mathrm{e}^{\frac{K_{+}}{2}}\left[a^{2}\left(\mathcal{P}^{1}-i \mathcal{P}^{2}\right)-\bar{b}^{2}\left(\mathcal{P}^{1}+i \mathcal{P}^{2}\right)-2 a \bar{b} \mathcal{P}^{3}\right]
$$

This form, however, carries redundant information arising from spin $3 / 2$ multiplets which should not appear in an ordinary $\mathcal{N}=1$ supergravity. We define a variable $\widetilde{\psi}_{\mu}=b \psi_{1 \mu}-\bar{a} \psi_{2 \mu}$ which is orthogonal to the ordinary gravitino $\psi_{\mu}$ in order that the fermion $\widetilde{\psi}_{\mu}$ would be a component of the spin $3 / 2$ multiplet. Imposing the invariance on the supersymmetry variation $\delta \widetilde{\psi}_{\mu}=0$, we obtain [15]

$$
0=\mathrm{e}^{\frac{K_{+}}{2}}\left[a b\left(\mathcal{P}^{1}-i \mathcal{P}^{2}\right)+\bar{a} \bar{b}\left(\mathcal{P}^{1}+i \mathcal{P}^{2}\right)+c_{-} \mathcal{P}^{3}\right]
$$

\footnotetext{
${ }^{8}$ Our notation differs from that of [15] by a sign, i.e., $e_{\mathrm{RR} A}$ in [15] becomes $-e_{\mathrm{RR} A}$, etc.
} 
Substituting (C.8) into (C.7), we write down the correct form of the superpotential

$$
\mathrm{e}^{\frac{K}{2}} \mathcal{W}=\frac{i}{4 \bar{a} b} \mathrm{e}^{\frac{K_{+}}{2}}\left[a b\left(\mathcal{P}^{1}-i \mathcal{P}^{2}\right)-\bar{a} \bar{b}\left(\mathcal{P}^{1}+i \mathcal{P}^{2}\right)-\mathcal{P}^{3}\right]
$$

Here we have to discuss the four-dimensional $\mathcal{N}=1$ Kähler potential $K$ in the left-hand side in (C.9). In terms of the four-dimensional dilaton $\varphi$, the function $K$ is defined as [11]

$$
K=K_{+}+4 \varphi=-\log i\left(\bar{X}^{A} \mathcal{F}_{A}-X^{A} \overline{\mathcal{F}}_{A}\right)+4 \varphi
$$

There is a relation among the ten-dimensional dilaton $\phi$, the Kähler potentials $K_{ \pm}$and the fourdimensional dilaton $\varphi$ as $\mathrm{e}^{-K_{ \pm}}=8 \mathrm{e}^{-2 \varphi+2 \phi}[15]$. We assumed that $\phi$ does not depend on the internal coordinates. Substituting (C.2) and (C.10) into (C.9), we rewrite the superpotential $\mathcal{W}$ as ${ }^{9}$

$$
\begin{aligned}
\mathcal{W} & =\frac{i}{4 \bar{a} b} \int_{\mathcal{M}}\left\langle\Phi_{+}, \frac{1}{\sqrt{2}} G^{\mathrm{f}}+\mathcal{D} \Pi_{-}\right\rangle \\
\Pi_{-} & \equiv \frac{1}{\sqrt{2}} A+i \operatorname{Im}\left(\mathcal{C} \Phi_{-}\right), \quad \mathcal{C} \equiv \sqrt{2} a b \mathrm{e}^{-\phi}=4 a b \mathrm{e}^{\frac{K_{-}}{2}-\varphi} .
\end{aligned}
$$

Here $\mathcal{C}$ is called a compensator of the dilaton $\phi$ (or $\varphi$ with the Kähler potential $K_{-}$). This is introduced to gauge away scale symmetry of the Weyl spinor $\Phi_{-}[15]$. (The spaces of the spinors $\Phi_{ \pm}$are the special Kähler geometry of local type [12].) Using the compensator $\mathcal{C}$, we rewrite the four-dimensional dilaton $\varphi$ as

$$
\begin{aligned}
\mathrm{e}^{-2 \varphi} & =\frac{|\mathcal{C}|^{2}}{16|a|^{2}|b|^{2}} \mathrm{e}^{-K_{-}}=\frac{i}{16|a|^{2}|b|^{2}} \int_{\mathcal{M}}\left\langle\mathcal{C} \Phi_{-}, \overline{\mathcal{C} \Phi_{-}}\right\rangle \\
& =\frac{1}{8|a|^{2}|b|^{2}}\left[\operatorname{Im}\left(\mathcal{C} Z^{I}\right) \operatorname{Re}\left(\mathcal{C} \mathcal{G}_{I}\right)-\operatorname{Re}\left(\mathcal{C} Z^{I}\right) \operatorname{Im}\left(\mathcal{C} \mathcal{G}_{I}\right)\right]
\end{aligned}
$$

We also rewrite the following function in terms of the basis forms and the flux charges:

$$
\frac{1}{\sqrt{2}} G^{\mathrm{fl}}+\mathcal{D} \Pi_{-} \sim\left(m_{\mathrm{RR}}^{A}+U^{I} p_{I}^{A}-\widetilde{U}_{I} q^{I A}\right) \omega_{A}-\left(e_{\mathrm{RR} A}-U^{I} e_{I A}+\widetilde{U}_{I} m_{A}{ }^{I}\right) \widetilde{\omega}^{A} .
$$

It is useful to introduce

$$
U^{I} \equiv \xi^{I}+i \operatorname{Im}\left(\mathcal{C} Z^{I}\right), \quad \widetilde{U}_{I} \equiv \widetilde{\xi}_{I}+i \operatorname{Im}\left(\mathcal{C} \mathcal{G}_{I}\right)
$$

Performing the integral in the superpotential (C.11a), we obtain the following form in the language of $\mathcal{N}=2$ theory:

$$
\mathcal{W}=-\frac{i}{4 \bar{a} b}\left[X^{A}\left(e_{\mathrm{RR} A}-U^{I} e_{I A}+\widetilde{U}_{I} m_{A}^{I}\right)-\mathcal{F}_{A}\left(m_{\mathrm{RR}}^{A}+U^{I} p_{I}^{A}-\widetilde{U}_{I} q^{I A}\right)\right] .
$$

Later we truncate $\mathcal{N}=2$ supersymmetry and reduce physical degrees of freedom.

\footnotetext{
${ }^{9}$ We used the same expressions of the real and the imaginary part of $\mathcal{C} \Phi$ - as in [15].
} 
In a similar way we evaluate an explicit form of the D-term from the supersymmetry variation of the gaugino. The supersymmetry truncation yields the $\mathcal{N}=1$ gaugino $\chi^{A}$ as a linear combination of the $\mathcal{N}=2$ gauginos $\chi^{a \mathcal{B}}$ as $\chi^{A}=-2 \mathrm{e}^{\frac{K_{+}}{2}} D_{b} X^{A}\left(\bar{n}^{\mathcal{A}} \epsilon_{\mathcal{A B}} \chi^{a \mathcal{B}}\right)$. Performing $\mathcal{N}=1$ supersymmetry variation and comparing a generic form of the $\mathcal{N}=1$ supersymmetry transformation rule given by $\delta \chi^{A}=\operatorname{Im} F_{\mu \nu}^{A} \gamma^{\mu \nu} \varepsilon+i D^{A} \varepsilon$, we obtain an explicit form of the D-term $D^{A}$ in the $\mathcal{N}=2$ language [15]:

$$
\begin{aligned}
D^{A}=\mathrm{e}^{2 \varphi} & \left(\left[(\operatorname{Im} \mathcal{N})^{-1}\right]^{A B}+2 \mathrm{e}^{K_{+}} \bar{X}^{A} X^{B}\right) \\
\times & \operatorname{Re}\left(\mathcal{C} Z^{I}\right)\left[e_{I B}+\mathcal{N}_{B C} p_{I}{ }^{C}\right]-\operatorname{Re}\left(\mathcal{C} \mathcal{G}_{I}\right)\left[m_{B}{ }^{I}+\mathcal{N}_{B C} q^{I C}\right] \\
& \left.\quad+c_{-}\left[\left(e_{\mathrm{RR} B}-\xi^{I} e_{I B}+\widetilde{\xi}_{I} m_{B}{ }^{I}\right)-\mathcal{N}_{B C}\left(m_{\mathrm{RR}}^{C}+\xi^{I} p_{I}{ }^{C}-\widetilde{\xi}_{I} q^{I C}\right)\right]\right\}
\end{aligned}
$$

\section{Orientifold projection}

It is necessary to introduce orientifold planes lying on the internal space in order to realize the tadpole cancellation and to evade a no-go theorem ${ }^{10}$ [52]. Due to the existence of the orientifold planes, the number of the supersymmetry parameters and physical degrees of freedom are truncated. This procedure is called the $\mathrm{O} 6$ orientifold projection. The orientifold projection affects the coefficients $a$ and $b$ in (A.1) as (see, for instance, $[12,45]$ )

$$
a=\bar{b} \mathrm{e}^{i \theta}, \quad|a|^{2}=|b|^{2}=\frac{1}{2},
$$

where $\theta$ is an arbitrary phase parameter.

The scalar components of vector multiplets and hypermultiplets in $\mathcal{N}=2$ supergravity are governed by the special Kähler geometry and the quaternionic geometry, respectively [17]. In type IIA theory compactified on Calabi-Yau three-fold, the former (latter) geometry is described by the moduli space of the Kähler form (the complex structure). In the theory compactified on generalized geometry, such two geometries are given by the spaces $\mathcal{M}_{ \pm}$discussed in the previous appendices [11,15]. Let us specify the supersymmetry truncation from $\mathcal{N}=2$ to $\mathcal{N}=1$ via the $\mathrm{O} 6$ orientifold projection on generalized geometries [15]. To preserve half of the supersymmetry, we set $a=\bar{b} \mathrm{e}^{i \theta}$ as in (D.1) and project out some physical degrees of freedom:

$$
\xi^{\hat{I}}=0=\operatorname{Im}\left(\mathcal{C} Z^{\hat{I}}\right)=\operatorname{Re}\left(\mathcal{C} \mathcal{G}_{\hat{I}}\right), \quad \widetilde{\xi}_{\check{I}}=0=\operatorname{Re}\left(\mathcal{C} Z^{\check{I}}\right)=\operatorname{Im}\left(\mathcal{C} \mathcal{G}_{\check{I}}\right),
$$

where the indices $I=0,1, \ldots, b^{-}$are split into $I=(\hat{I}, \check{I})$. Due to this, each $\mathcal{N}=2$ hypermultiplet in type IIA is decomposed into two $\mathcal{N}=1$ chiral multiplets with opposite spins. In addition, $\mathcal{N}=2$ vector multiplets with indices $A=(0, a)$ are truncated as

$$
A_{\mu}^{\check{A}}=0, \quad X^{\hat{A}}=0,
$$

\footnotetext{
${ }^{10}$ We do not analyze the Bianchi identities themselves in this paper. A detailed discussions can be found, for instance, in $[12]$.
} 


$$
\mathcal{F}_{\hat{A}}=0, \quad \mathcal{N}_{\check{A} \hat{B}}=0 ; \quad\left(K_{+}\right)_{\check{a} \hat{b}}=0, \quad D_{\check{a}} X^{\hat{B}}=D_{\hat{a}} X^{\check{B}}=0 .
$$

Note that one has to truncate out the graviphoton $A_{\mu}^{0}$. We split the indices $A=0,1, \ldots, n_{v}$ (where $n_{v}=b^{+}$) as $\check{A}=0,1, \ldots, n_{c h}$ and $\hat{A}=1, \ldots, \hat{n}_{v}=n_{v}-n_{c h}$ (with a restriction $n_{v} \geq n_{c h}$ ). This means that $\mathcal{N}=2$ vector multiplets are decomposed into $\mathcal{N}=1$ vector multiplets and chiral multiplets with respective numbers $n_{v}$ : Some degrees of freedom are projected out in such a way as $n_{v} \rightarrow \hat{n}_{v}$ in the vector multiplets, and as $n_{v} \rightarrow n_{c h}$ in the chiral multiplets. Imposing (D.2a) and (D.2b) on $\Phi_{+}$and on $\Pi_{-}$, we obtain $\Phi_{+}=X^{\check{A}} \omega_{\check{A}}-\mathcal{F}_{\check{A}} \widetilde{\omega}^{\check{A}}$ and $\Pi_{-}=U^{\check{I}} \alpha_{\check{I}}-\widetilde{U}_{\hat{I}} \beta^{\hat{I}}$, respectively. Substituting them into the previous results, we write down the reduced functions:

$$
\begin{aligned}
\mathcal{W} & =-\frac{i}{4 \bar{a} b}\left[X^{\check{A}}\left(e_{\mathrm{RR} \check{A}}-U^{\check{I}} e_{\check{I} \check{A}}+\widetilde{U}_{\hat{I}} m_{\check{A}}{ }^{\hat{I}}\right)-\mathcal{F}_{\check{A}}\left(m_{\mathrm{RR}}^{\check{A}}+U^{\check{I}} p_{\check{I}}^{\check{A}}-\widetilde{U}_{\hat{I}} q^{\hat{I} \check{A}}\right)\right], \\
K & =K_{+}+4 \varphi \\
K_{+} & =-\log i\left(\bar{X}^{\check{A}} \mathcal{F}_{\check{A}}-X^{\check{A}} \overline{\mathcal{F}}_{\check{A}}\right), \\
\mathrm{e}^{-2 \varphi} & =\frac{1}{2}\left[\operatorname{Im}\left(\mathcal{C} Z^{\check{I}}\right) \operatorname{Re}\left(\mathcal{C} \mathcal{G}_{\check{I}}\right)-\operatorname{Re}\left(\mathcal{C} Z^{\hat{I}}\right) \operatorname{Im}\left(\mathcal{C} \mathcal{G}_{\hat{I}}\right)\right] .
\end{aligned}
$$

Substituting the truncation rules (D.2a) and (D.2b) into (C.16) with setting $D^{A} \rightarrow D^{\hat{A}}$, we also obtain the D-term in $\mathcal{N}=1$ theory as

$$
D^{\hat{A}}=\mathrm{e}^{2 \varphi}\left[(\operatorname{Im} \mathcal{N})^{-1}\right]^{\hat{A} \hat{B}}\left\{\operatorname{Re}\left(\mathcal{C} Z^{\hat{I}}\right)\left[e_{\hat{I} \hat{B}}+\mathcal{N}_{\hat{B} \hat{C}} p_{\hat{I}}^{\hat{C}}\right]-\operatorname{Re}\left(\mathcal{C} \mathcal{G}_{\check{I}}\right)\left[m_{\hat{B}}^{\check{I}}+\mathcal{N}_{\hat{B} \hat{C}} q^{\check{I} \hat{C}}\right]\right\} .
$$

We should notice that the D-term (D.3e) is a complex because of the existence of an (anti-)holomorphic function $\mathcal{N}_{\hat{B} \hat{C}}$. This appears in [17,54]. This situation generically occurs when complex forms of flux variables are turned on. Then we should carefully define the scalar potential from this D-term. We also substituted $c_{-}=|a|^{2}-|b|^{2}=0$ by the O6 orientifold projection [15].

\section{References}

[1] P. Candelas, G.T. Horowitz, A. Strominger and E. Witten, "Vacuum configurations for superstrings," Nucl. Phys. B 258 (1985) 46.

[2] A. Strominger, "Superstrings with torsion," Nucl. Phys. B 274 (1986) 253.

[3] S. Chiossi and S. Salamon, "The intrinsic torsion of $S U(3)$ and $G_{2}$ structures," Differential Geometry, Valencia 2001, World Sci. Publishing (2002) 115 [arXiv:math/0202282].

[4] J.P. Gauntlett, D. Martelli and D. Waldram, "Superstrings with intrinsic torsion," Phys. Rev. D 69 (2004) 086002 [arXiv:hep-th/0302158].

[5] N.J. Hitchin, "Generalized Calabi-Yau manifolds," Quart. J. Math. Oxford Ser. 54 (2003) 281 [arXiv:math/0209099].

M. Gualtieri, "Generalized complex geometry," Oxford Univ. DPhil thesis [arXiv:math/0401221]. 
[6] M. Graña, R. Minasian, M. Petrini and A. Tomasiello, "Supersymmetric backgrounds from generalized Calabi-Yau manifolds," JHEP 0408 (2004) 046 [arXiv:hep-th/0406137]; "Generalized structures of $\mathcal{N}=1$ vacua," JHEP 0511 (2005) 020 [arXiv:hep-th/0505212].

[7] M. Graña, J. Louis and D. Waldram, "Hitchin functionals in $\mathcal{N}=2$ supergravity," JHEP 0601 (2006) 008 [arXiv:hep-th/0505264]; "SU(3) $\times S U(3)$ compactification and mirror duals of magnetic fluxes," JHEP 0704 (2007) 101 [arXiv:hep-th/0612237].

[8] P. Koerber, "Stable D-branes, calibrations and generalized Calabi-Yau geometry," JHEP 0508 (2005) 099 [arXiv:hep-th/0506154].

[9] L. Martucci and P. Smyth, "Supersymmetric D-branes and calibrations on general $\mathcal{N}=1$ backgrounds," JHEP 0511 (2005) 048 [arXiv:hep-th/0507099].

[10] M. Graña, "Flux compactifications in string theory: A comprehensive review," Phys. Rept. 423 (2006) 91 [arXiv:hep-th/0509003].

[11] I. Benmachiche and T.W. Grimm, "Generalized $\mathcal{N}=1$ orientifold compactifications and the Hitchin functionals," Nucl. Phys. B 748 (2006) 200 [arXiv:hep-th/0602241].

[12] M. Graña, R. Minasian, M. Petrini and A. Tomasiello, "A scan for new $\mathcal{N}=1$ vacua on twisted tori," JHEP 0705 (2007) 031 [arXiv:hep-th/0609124].

[13] A. Micu, E. Palti and G. Tasinato, "Towards Minkowski vacua in type II string compactifications," JHEP 0703 (2007) 104 [arXiv:hep-th/0701173].

[14] P. Koerber and L. Martucci, "From ten to four and back again: how to generalize the geometry," JHEP 0708 (2007) 059 [arXiv:0707.1038 [hep-th]].

[15] D. Cassani and A. Bilal, "Effective actions and $\mathcal{N}=1$ vacuum conditions from $S U(3) \times S U(3)$ compactifications," JHEP 0709 (2007) 076 [arXiv:0707.3125 [hep-th]].

[16] M. Graña, R. Minasian, M. Petrini and D. Waldram, "T-duality, generalized geometry and nongeometric backgrounds," JHEP 0904 (2009) 075 [arXiv:0807.4527 [hep-th]].

[17] L. Andrianopoli, M. Bertolini, A. Ceresole, R. D'Auria, S. Ferrara, P. Fré and T. Magri, “ $\mathcal{N}=2$ supergravity and $\mathcal{N}=2$ super Yang-Mills theory on general scalar manifolds: Symplectic covariance, gaugings and the momentum map," J. Geom. Phys. 23 (1997) 111 [arXiv:hep-th/9605032].

[18] N. Kaloper and R.C. Myers, "The O(dd) story of massive supergravity," JHEP 9905 (1999) 010 [arXiv:hep-th/9901045].

[19] G. Dall'Agata and S. Ferrara, "Gauged supergravity algebras from twisted tori compactifications with fluxes," Nucl. Phys. B 717 (2005) 223 [arXiv:hep-th/0502066]. 
[20] C.M. Hull and R.A. Reid-Edwards, "Flux compactifications of string theory on twisted tori," arXiv:hep-th/0503114.

[21] S. Kachru, M.B. Schulz, P.K. Tripathy and S.P. Trivedi, "New supersymmetric string compactifications," JHEP 0303 (2003) 061 [arXiv:hep-th/0211182].

[22] J. Shelton, W. Taylor and B. Wecht, "Nongeometric flux compactifications," JHEP 0510 (2005) 085 [arXiv:hep-th/0508133].

[23] C.M. Hull, "A geometry for non-geometric string backgrounds," JHEP 0510 (2005) 065 [arXiv:hep-th/0406102].

[24] C.M. Hull and R.A. Reid-Edwards, "Gauge symmetry, T-duality and doubled geometry," JHEP 0808 (2008) 043 [arXiv:0711.4818 [hep-th]].

[25] D. Lüst and D. Tsimpis, "Supersymmetric $\mathrm{AdS}_{4}$ compactifications of IIA supergravity," JHEP 0502 (2005) 027 [arXiv:hep-th/0412250].

[26] G. Lopes Cardoso, G. Curio, G. Dall'Agata, D. Lüst, P. Manousselis and G. Zoupanos, "NonKähler string backgrounds and their five torsion classes," Nucl. Phys. B 652 (2003) 5 [arXiv:hepth/0211118].

[27] G. Lopes Cardoso, G. Curio, G. Dall'Agata and D. Lüst, "Heterotic string theory on nonKaehler manifolds with H-flux and gaugino condensate," Fortsch. Phys. 52 (2004) 483 [arXiv:hepth/0310021].

[28] B.S. Acharya, F. Benini and R. Valandro, "Fixing moduli in exact type IIA flux vacua," JHEP 0702 (2007) 018 [arXiv:hep-th/0607223].

[29] P. Koerber, D. Lüst and D. Tsimpis, "Type IIA AdS 4 compactifications on cosets, interpolations and domain walls," JHEP 0807 (2008) 017 [arXiv:0804.0614 [hep-th]].

[30] C. Caviezel, P. Koerber, S. Körs, D. Lüst, D. Tsimpis and M. Zagermann, "The effective theory of type IIA $\mathrm{AdS}_{4}$ compactifications on nilmanifolds and cosets," Class. Quant. Grav. 26 (2009) 025014 [arXiv:0806.3458 [hep-th]].

[31] S. Ferrara, R. Kallosh and A. Strominger, "N $=2$ extremal black holes," Phys. Rev. D 52 (1995) 5412 [arXiv:hep-th/9508072].

[32] S. Ferrara and R. Kallosh, "Supersymmetry and attractors," Phys. Rev. D 54 (1996) 1514 [arXiv:hep-th/9602136].

[33] S. Ferrara, G.W. Gibbons and R. Kallosh, "Black holes and critical points in moduli space," Nucl. Phys. B 500 (1997) 75 [arXiv:hep-th/9702103]. 
[34] R. Kallosh, N. Sivanandam and M. Soroush, "The non-BPS black hole attractor equation," JHEP 0603 (2006) 060 [arXiv:hep-th/0602005]; "Exact attractive non-BPS STU black holes," Phys. Rev. D 74 (2006) 065008 [arXiv:hep-th/0606263].

[35] J.P. Hsu, A. Maloney and A. Tomasiello, "Black hole attractors and pure spinors," JHEP 0609 (2006) 048 [arXiv:hep-th/0602142].

[36] A. Ceresole and G. Dall'Agata, "Flow equations for non-BPS extremal black holes," JHEP 0703 (2007) 110 [arXiv:hep-th/0702088].

[37] D. Astefanesei and H. Yavartanoo, "Stationary black holes and attractor mechanism," Nucl. Phys. B 794 (2008) 13 [arXiv:0706.1847 [hep-th]].

[38] S.B. Giddings, S. Kachru and J. Polchinski, "Hierarchies from fluxes in string compactifications," Phys. Rev. D 66 (2002) 106006 [arXiv:hep-th/0105097].

[39] A. Giryavets, S. Kachru, P.K. Tripathy and S.P. Trivedi, "Flux compactifications on Calabi-Yau threefolds," JHEP 0404 (2004) 003 [arXiv:hep-th/0312104].

[40] O. DeWolfe, A. Giryavets, S. Kachru and W. Taylor, "Type IIA moduli stabilization," JHEP 0507 (2005) 066 [arXiv:hep-th/0505160].

[41] R. Kallosh, "New attractors," JHEP 0512 (2005) 022 [arXiv:hep-th/0510024].

[42] G. Dall'Agata, "Non-Kähler attracting manifolds," JHEP 0604 (2006) 001 [arXiv:hepth/0602045].

[43] S. Bellucci, S. Ferrara, R. Kallosh and A. Marrani, "Extremal black hole and flux vacua attractors," to appear in the proceedings of Winter School on Attractor Mechanism (SAM 2006), Frascati, Italy, 20-24 Mar 2006, Lect. Notes Phys. 755 (2008) 115 [arXiv:0711.4547 [hep-th]].

[44] L. Anguelova, "Flux vacua attractors and generalized compactifications," JHEP 0901 (2009) 017 [arXiv:0806.3820 [hep-th]].

[45] T.W. Grimm and J. Louis, "The effective action of type IIA Calabi-Yau orientifolds," Nucl. Phys. B 718 (2005) 153 [arXiv:hep-th/0412277].

T.W. Grimm, "The effective action of type II Calabi-Yau orientifolds," Fortsch. Phys. 53 (2005) 1179 [arXiv:hep-th/0507153].

[46] B. de Carlos, S. Gurrieri, A. Lukas and A. Micu, "Moduli stabilisation in heterotic string compactifications," JHEP 0603 (2006) 005 [arXiv:hep-th/0507173].

[47] P. Breitenlohner and D.Z. Freedman, "Positive energy in Anti-de Sitter backgrounds and gauged extended supergravity," Phys. Lett. B 115 (1982) 197. 
[48] T. Kimura and P. Yi, "Comments on heterotic flux compactifications," JHEP 0607 (2006) 030 [arXiv:hep-th/0605247].

[49] E. Palti, "Low energy supersymmetry from non-geometry," JHEP 0710 (2007) 011 [arXiv:0707.1595 [hep-th]].

[50] G. Dall'Agata, N. Prezas, H. Samtleben and M. Trigiante, "Gauged supergravities from twisted doubled tori and non-geometric string backgrounds," Nucl. Phys. B 799 (2008) 80 [arXiv:0712.1026 [hep-th]].

[51] T. Kimura, "Index theorems on torsional geometries," JHEP 0708 (2007) 048 [arXiv:0704.2111 [hep-th]].

[52] J.M. Maldacena and C. Nuñez, "Supergravity description of field theories on curved manifolds and a no go theorem," Int. J. Mod. Phys. A 16 (2001) 822 [arXiv:hep-th/0007018].

[53] E. Bergshoeff, R. Kallosh, T. Ortin, D. Roest and A. Van Proeyen, "New formulations of $D=$ 10 supersymmetry and D8-O8 domain walls," Class. Quant. Grav. 18 (2001) 3359 [arXiv:hepth/0103233].

[54] T.R. Taylor and C. Vafa, "RR flux on Calabi-Yau and partial supersymmetry breaking," Phys. Lett. B 474 (2000) 130 [arXiv:hep-th/9912152].

R. D'Auria, S. Ferrara, M. Trigiante and S. Vaula, " $\mathcal{N}=1$ reductions of $\mathcal{N}=2$ supergravity in the presence of tensor multiplets," JHEP 0503 (2005) 052 [arXiv:hep-th/0502219]. 